\title{
Differential Geometry of Gerbes and Differential Forms
}

\author{
Lawrence Breen* \\ Institut Galilée \\ Université Paris 13 \\ 99, avenue J. - B. Clément \\ 93430 Villetaneuse, France \\ breen@math.univ-paris13.fr
}

To Murray Gerstenhaber and Jim Stasheff

Summary. We discuss certain aspects of the combinatorial approach to the differential geometry of non-abelian gerbes due to W. Messing and the author [5], and give a more direct derivation of the associated cocycle equations. This leads us to a more restrictive definition than in [5] of the corresponding coboundary relations. We also show that the diagrammatic proofs of certain local curving and curvature equations may be replaced by computations with differential forms.

\section{Introduction}

It is a classical fact 1 that to a principal $G$-bundle $P$ on a scheme $X$, endowed with a connection $\epsilon$, is associated a Lie $(G)$-valued 2-form $\kappa$ on $P$, the curvature of the connection, satisfying a certain $G$-equivariance condition. While $\kappa$ does not in general descend to a 2 -form on $X$, the equivariance condition may be viewed as a descent condition for $\kappa$ from a 2 -form on $P$ to a 2 -form on $X$, but now with values in the Lie algebra of the gauge group $P^{\text {ad }}$ of $P$. The connection on $P$ also induces a connection $\mu$ on the group $P^{\text {ad }}$, and the 2form $\kappa$ satisfies the Bianchi equation, an equation which may be expressed in global terms as

$$
\mathrm{d} \kappa+[\mu, \kappa]=0
$$

(5. proposition 1.7, 4 theorem 3.7). Choosing a local trivialization of the bundle $P$, on an open cover $\mathcal{U}:=\coprod_{i \in I} U_{i}$ of $X$, the connection $\epsilon$ is described

\footnotetext{
* Unité Mixte de Recherche CNRS 7539

${ }^{1}$ at least in a differential geometric setting, see 9, but the same construction can be carried out within the context of algebraic geometry.
} 
by a family of Lie $(G)$-valued connection 1 -forms $\omega_{i}$ defined on the open sets $U_{i}$, and the associated curvature $\kappa$ corresponds to a family of Lie $(G)$-valued 2forms $\kappa_{i}$ defined, according to the so-called structural equation of Elie Cartan, by the formula

$$
\kappa_{i}=\mathrm{d} \omega_{i}+\frac{1}{2}\left[\omega_{i}, \omega_{i}\right]
$$

Equation (1.1) then reduces to the classical Bianchi identity

$$
\mathrm{d} \kappa_{i}+\left[\omega_{i}, \kappa_{i}\right]=0 .
$$

J. -L. Brylinski introduced in 7 the notions of connection $\epsilon$ and curving $K$ on an abelian $G$-gerbe $\mathcal{P}$ on a space $X$ (where $G$ was the multiplicative group $G_{m}$, or rather in his framework the group $U(1)$ ), and showed that to such connective data $(\epsilon, K)$ is associated a closed $G_{m}$-valued 3-form $\omega$ on $X$, the 3-curvature. More recently, W. Messing and the author extended these concepts in [5] from abelian to general, not necessarily abelian, gerbes $\mathcal{P}$ on a scheme $X$. The coefficients of such a gerbe no longer constitute a sheaf of groups as in the principal bundle situation, but rather a monoidal stack $\mathcal{G}$ on $X$, as is to be expected in that categorified setting. In particular, when the gerbe is associated to a given non-abelian group $G$ (so that we refer to it as a $G$ gerbe), the corresponding coefficient stack $\mathcal{G}$ is the monoidal stack associated to the prestack determined by the crossed module $G \longrightarrow \operatorname{Aut}(G)$, where $\operatorname{Aut}(G)$ is the sheaf of local automorphisms of $G$. It may also be described more invariantly as the monoidal stack of $G$-bitorsors on $X$. Once more, to the gerbe $\mathcal{P}$ is associated its gauge stack, a twisted form $\mathcal{P}^{\text {ad }}:=\mathcal{E} q(\mathcal{P}, \mathcal{P})$ of the given monoidal stack $\mathcal{G}$, and the connection on $\mathcal{P}$ induces a connection $\mu$ on $\mathcal{P}^{\text {ad }}$. By analogy with the principal bundle case, the corresponding 3 curvature $\Omega$, viewed as a global 3-form on $X$, now takes its values in the arrows of the stack $\mathcal{P}^{\text {ad }}$.

There now arises a new, and at first sight somewhat surprising feature, but which is simply another facet of the categorification context in which we are operating. The 3 -form $\Omega$ is accompanied by an auxiliary 2 -form $\kappa$ with values in the objects of the gauge stack $\mathcal{P}^{\text {ad }}$, which we called in [5] the fake curvature of the given connective structure $(\epsilon, K)$. A first relation between the forms $\Omega$ and $\kappa$ comes from the very definition [5] (4.1.20), (4.1.22) of $\Omega$, and may be stated as in 5 (4.3.8) as the categorical equation

$$
t \Omega+\mathrm{d} \kappa+[\mu, \kappa]=0
$$

where $t$ stands for "target" of a 1-arrow with source the identity object $I$ in the stack of $\operatorname{Lie}\left(\mathcal{P}^{\text {ad }}\right)$-valued 3 -forms on $X$. On the other hand, the 3 -form $\Omega$ is

\footnotetext{
${ }^{2}$ The canonical divided power $1 / 2[\omega, \omega]$ of the 2 -form $[\omega, \omega]$ is also denoted $\omega \wedge \omega$ or $[\omega]^{(2)}$.
} 
no longer closed, even in the $\mu$-twisted sense described for principal bundles by (1.1). It satisfies instead the following more complicated analogue [5] (4.1.33) of the Bianchi identity (1.1):

$$
\mathrm{d} \Omega+[\mu, \Omega]+[\mathcal{K}, \kappa]=0 .
$$

While the first two terms in this equation are similar to those of (1.1), the categorification term $\mathcal{K}$ is an arrow in the stack of 2 -forms with values in the monoidal stack $\mathcal{E} q\left(\mathcal{P}^{\text {ad }}, \mathcal{P}^{\text {ad }}\right)$ induced by the curving $K$. The pairing of $\mathcal{K}$ with $\kappa$ is induced by the evaluation of the natural transformation $\mathcal{K}$ between functors from $\mathcal{P}^{\text {ad }}$ to itself on the object $\kappa$ of $\mathcal{P}^{\text {ad }}$.

The price to be paid for the compact form in which the global curvature equations (1.4) and (1.5) have been stated is their rather abstract nature, and it is of interest to describe them in a more local form in terms of traditional group-valued differential forms, just as was done in (1.3) for equation (1.1). Such a local description was already obtained in [5], both for the cocycle conditions (1.4) and (1.5), and for the corresponding coboundary equations which arise when alternate local trivializations of the gerbe have been chosen. However, the determination of those local equations was rather indirect, as it required a third description of a gerbe, which we have called the semilocal description [6] $\S 4$, and which has also appeared elsewhere in a various situations [18, [14, 8].

The present text may be viewed as a companion piece to the author's [6]. Its main purpose is to provide a more transparent construction than in [5] of the cocycle conditions and related equations associated to a gerbe with curving data summarized in [5] theorem 6.4. We restrict our attention, as in [6], to gerbes which are connected rather than locally connected, as these determine Čech cohomology classes. A cocyclic description in the general case requires hypercovers and could be dealt with along the lines discussed in [3], but would not shed any additional light on the phenomena being investigated here. Our main results are to be found in sections 4 and 5 , while section 3 reviews for the reader's convenience some aspects of [5] and 6. Section 2 is a review of some of the formulas in the differential calculus of Lie $(G)$-valued forms, a few of which do not appear to be well-known.

Another aim of the present work is to revisit the quite complicated coboundary equations of $[5, \S 6.2$. The coboundary equations which arise here are simpler, and more consistent than those of $[5]$ with a non-abelian Čech-de Rham interpretation. We refer to remark 5.1 for a specific comparison between the two notions. In order to make this comparison easier, we have chosen the orientations of our arrows consistenly with $[5$. This accounts for example for the strange choice of orientation of the arrow $B_{i}$ in diagram (4.13), or for the change of sign (4.28) for the arrow $\gamma_{i j}$. 
A final purpose of this text is to explain how the diagrammatic proofs of some of the local results of [5] can be replaced by more classical computations involving Lie $(G)$-valued differential forms. For this reason, we have given two separate computations for certain equations, one diagrammatic and the other classical. We do not assert that one of the two methods of proof is always preferable, though one might contend that diagrams provide a better understanding of the situation than the corresponding manipulation of differential forms. As the level of categorification increases, so will the dimension of the diagrams to be considered, and it may not be realistic to expect to tread along the diagrammatic path much beyond the hypercube proof [5] (4.1.33) of the higher Bianchi equation (1.5). The generality and algebraicity of the formalism of differential forms must then come into its own. In addition, it is our hope that the present approach, which extends to the gerbe context the traditional methods of differential geometry, will provide an accessible point of entry into this topic. A number of other authors have recently described certain aspects of the differential geometry of gerbes in terms of differential forms, particularly [1, 12, and [16, 2].

I wish to thank Bernard Julia and Camille Laurent-Gengoux for enlightening discussions on related topics. The impetus for the present work was provided by my collaboration with Wiliam Messing on our joint papers [4] and 5 . It is a pleasure to thank him here for our instructive and wide-ranging discussions over all these years.

\section{Group-valued differential forms}

\section{1}

Let $X$ be an $S$-scheme. We assume from now on for simplicity that that the primes 2 and 3 are invertible in the ring of functions of $S$ (for example $S=\operatorname{Spec}(k)$ where $k$ is a field of characteristic $\neq 2,3)$. A relative differential $n$-form on an $S$-scheme $X$, with values in a sheaf of $\mathcal{O}_{S}$-Lie algebras $\mathfrak{g}$ is defined as a global section of the sheaf $\mathfrak{g} \otimes_{\mathcal{O}_{S}} \Omega_{X / S}^{n}$ on $X$. When $X / S$ is smooth,

$$
\mathfrak{g} \otimes_{\mathcal{O}_{S}} \Omega_{X / S}^{n} \simeq \operatorname{Hom}_{\mathcal{O}_{X}}\left(T_{X / S}^{n}, \mathfrak{g}_{X}\right)
$$

where $\mathfrak{g}_{X}:=\mathfrak{g} \otimes_{\mathcal{O}_{S}} \mathcal{O}_{X}$ and $T_{X / S}^{n}$ is the $n$-th exterior power $\wedge^{n} T_{X / S}$ of the relative tangent sheaf $T_{X / S}$, i.e the sheaf of relative $n$-vector fields on $X$. Such an $n$-form is nothing else than an $\mathcal{O}_{X}$-linear map

$$
T_{X / S}^{n} \longrightarrow \mathfrak{g}_{X}
$$

In view of this definition, such a map is classically called a $\mathfrak{g}$-valued differential form. A more geometric description of such forms is given in [4, 
following the ideas of A. Kock in the context of synthetic differential geometry [10, 11]. It is based on the consideration, for any positive integer $n$, of the scheme $\Delta_{X / S}^{n}$ of relative infinitesimal $n$-simplexes on $X$. For any $S$ scheme $T$, a $T$-valued point of $\Delta_{X / S}^{n}$ consists of an $(n+1)$-tuple of $T$-valued points $\left(x_{0}, \ldots, x_{n}\right)$ of $X$ which are pairwise close to first order in an appropriate sense [4] (1.4.9). We view $\Delta_{X}^{n}$ as an $X$-scheme via the projection $p_{0}$ of such points to $x_{0}$. As $n$ varies, the schemes $\Delta_{X / S}^{n}$ determine a simplicial $X$-scheme $\Delta_{X / S}^{*}$, whose face and degeneracy operations are induced by the usual projection and injection morphisms $X^{n} \longrightarrow X^{n \pm 1}$.

Let $G$ be a flat $S$-group scheme, with $\mathcal{O}_{S}$-Lie algebra $\mathfrak{g}$. A relative $\mathfrak{g}$ valued $n$-form (2.2) on $X / S$ may then be identified by [4 proposition 2.5 with a morphism of $S$-schemes

$$
\Delta_{X / S}^{n} \stackrel{f}{\longrightarrow} G
$$

whose restriction to the degenerate subsimplex $s \Delta_{X / S}^{n}$ of $\Delta_{X / S}^{n}$ factors through the unit section of $G$. When differential forms are expressed in this combinatorial language, they deserve to be called $G$-valued differential forms, even though they actually coincide with the traditional $\mathfrak{g}$-valued differential forms (2.1), (2.2). In the combinatorial context, our notation will be multiplicative, and additive when we pass to the traditional language of differential forms.

We will now discuss some of the features of these $\mathfrak{g}$-valued forms, and refer to [4] for further discussion. First of all, let us recall that the action of the symmetric group $S_{n+1}$ on a combinatorial differential $n$-form $\omega\left(x_{0}, \ldots, x_{n}\right)$ by permutation of the variables is given by

$$
\omega\left(x_{\sigma(0)}, \ldots, x_{\sigma(n)}\right)=\omega\left(x_{0}, \ldots, x_{n}\right)^{\epsilon(\sigma)}
$$

where $\epsilon(\sigma)$ is the signature of $\sigma$. Also, the commutator pairing

$$
[g, h]:=g h g^{-1} h^{-1}
$$

on the group $G$ determines a bracket pairing on $\mathfrak{g}$-valued forms of degree $\geq 1$, defined combinatorially by the rule

$$
\left(\mathfrak{g} \otimes_{\mathcal{O}_{S}} \Omega_{X / S}^{m}\right) \times\left(\mathfrak{g} \otimes_{\mathcal{O}_{S}} \Omega_{X / S}^{n}\right) \quad \rightarrow \quad\left(\mathfrak{g} \otimes_{\mathcal{O}_{S}} \Omega_{X / S}^{m+n}\right)
$$

which sends $\left(\omega, \omega^{\prime}\right)$ to $\left[\omega, \omega^{\prime}\right]$, where

$$
\left[\omega, \omega^{\prime}\right]\left(x_{0}, \ldots, x_{m+n}\right):=\left[\omega\left(x_{0}, \ldots, x_{m}\right), \omega^{\prime}\left(x_{m}, \ldots, x_{m+n}\right)\right] .
$$

This pairing is defined in classical terms, by

$$
\left[\omega, \omega^{\prime}\right]:=\left[Y, Y^{\prime}\right] \otimes\left(\eta \wedge \eta^{\prime}\right)
$$


for any pair of forms $\omega:=Y \otimes \eta$ and $\omega^{\prime}:=Y^{\prime} \otimes \eta^{\prime}$ in $\mathfrak{g} \otimes_{\mathcal{O}_{S}} \Omega_{X / S}^{*}$. It endows $\mathfrak{g} \otimes_{\mathcal{O}_{S}} \Omega_{X / S}^{*}$ with the structure of a graded $\mathcal{O}_{S}$-Lie algebra. In particular, the bracket satisfies the graded commutativity rule

$$
[f, g]=(-1)^{|f||g|+1}[g, f],
$$

where $|f|$ is the degree of the form $f$, so that

$$
[f, f]=0
$$

whenever $|f|$ is even. The graded Jacobi identity is expressed (in additive notation) as:

$$
(-1)^{|f||h|}[f,[g, h]]+(-1)^{|f||g|}[g,[h, f]]+(-1)^{|g||h|}[h,[f, g]]=0 .
$$

In particular,

$$
[f,[f, f]]=0
$$

and, when $|f|=|g|=1$,

$$
\left[f, \frac{1}{2}[g, g]\right]=[[f, g], g] .
$$

Let $\operatorname{Aut}(G)$ be the sheaf of local automorphisms of $G$, whose group of

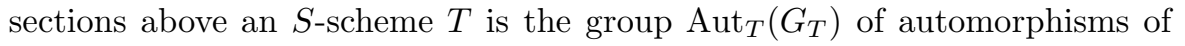
the $T$-group $G_{T}:=G \times_{S} T$. The definition (2.3) of a combinatorial $n$-form still makes sense when $G$ is replaced by a sheaf of groups $F$ on $S$, and the traditional description of such combinatorial $n$-forms as $n$-forms with values in the Lie algebra of $F$ remains valid by [4] proposition 2.3 when $F=\operatorname{Aut}(G)$. The evaluation map

$$
\begin{gathered}
\operatorname{Aut}(G) \times G \longrightarrow G \\
(u, g) \quad \mapsto u(g)
\end{gathered}
$$

induces for all pair of positive integers a bilinear pairing

$$
\left(\operatorname{Lie}(\operatorname{Aut}(G)) \otimes_{\mathcal{O}_{S}} \Omega^{m}\right) \times\left(\mathfrak{g} \otimes_{\mathcal{O}_{S}} \Omega_{X / S}^{n}\right) \rightarrow\left(\mathfrak{g} \otimes_{\mathcal{O}_{S}} \Omega_{X / S}^{m+n}\right)
$$

which sends $(u, g)$ to $[u, g]$, where

$[u, g]\left(x_{0}, \ldots, x_{m+n}\right):=u\left(x_{0}, \ldots, x_{m}\right)\left(g\left(x_{m}, \ldots, x_{m+n}\right)\right) g\left(x_{m}, \ldots, x_{m+n}\right)^{-1}$.

This pairing is compatible with the pairings (2.4) associated to the $S$-groups $G$ and $\operatorname{Aut}(G)$ in the following sense. For any pair of $\mathfrak{g}$-valued forms $g, g^{\prime}$, and an $\operatorname{Aut}(G)$-valued form $u$,

$$
\left[i(g), g^{\prime}\right]=\left[g, g^{\prime}\right] \quad \text { and } \quad i([u, g])=[u, i(g)]
$$

where $i: G \longrightarrow \operatorname{Aut}(G)$ is the inner conjugation map $i(\gamma)(g):=\gamma g \gamma^{-1}$. More generally, an isomorphism $r: G \longrightarrow G^{\prime}$ induces a morphism $r$ from $G$-valued 
combinatorial $n$-forms to $G^{\prime}$-valued combinatorial $n$-forms, compatible with the Lie bracket operation (2.4), and which corresponds in classical terms to the morphism Lie $(r) \otimes_{o s c} 1: \mathfrak{g} \otimes_{\mathcal{O}_{S}} \Omega_{X / S}^{n} \longrightarrow \mathfrak{g}^{\prime} \otimes \Omega_{X / S}^{n}$. The functoriality of the bracket (2.7) is expressed by the formula

$$
r\left([u, g]=\left[{ }^{r} u, r(g)\right]\right.
$$

where ${ }^{r} u:=r u r^{-1}$.

When $u$ is an $\operatorname{Aut}(G)$-valued form of degree $m \geq 1$ and $g$ is a $G$-valued function, the definition of a pairing

$$
\begin{gathered}
\left(\text { Lie } \operatorname{Aut}(G) \otimes_{\mathcal{O}_{S}} \Omega_{X / S}^{m}\right) \times G \\
(u, g) \\
\mapsto \quad \mathfrak{g} \otimes_{\mathcal{O}_{S}} \Omega_{X / S}^{m} \\
\mapsto u, g]
\end{gathered}
$$

is still given by the formula (2.8), but now with $n=0$. This pairing are no longer linear in $g$, but instead satisfies the equation

$$
\left[u, g g^{\prime}\right]=[u, g]+{ }^{g}\left[u, g^{\prime}\right]
$$

where for any $G$-valued form $\omega$ and any $G$-valued function $g$ the adjoint left action ${ }^{g} \omega$ of a function $g$ on a form $\omega$ is defined combinatorially by

$$
\left({ }^{g} \omega\right)\left(x_{0}, \ldots, x_{n}\right):=g\left(x_{0}\right) \omega\left(x_{0}, \ldots, x_{n}\right) g\left(x_{0}\right)^{-1},
$$

(and this expression is in fact equal to $g\left(x_{i}\right) \omega\left(x_{0}, \ldots, x_{n}\right) g\left(x_{i}\right)^{-1}$ for any $0 \leq i \leq n)$. In classical notation this corresponds, for $\omega=Y \otimes \eta \in \mathfrak{g} \otimes \Omega_{X / S}^{n}$, to the formula

$$
{ }^{g}(Y \otimes \eta)={ }^{g} Y \otimes \eta
$$

for the adjoint left action of $g$ on $Y$. The adjoint right action $\omega^{\gamma}$ is defined by

$$
\omega^{g}:={ }^{\left(g^{-1}\right)} \omega
$$

so that

$$
\omega^{g}\left(x_{0}, \ldots, x_{n}\right)=g\left(x_{0}\right)^{-1} \omega\left(x_{0}, \ldots, x_{n}\right) g\left(x_{0}\right) .
$$

Similarly, when $g$ is a $G$-valued and $u$ an $\operatorname{Aut}(G)$-valued form, a pairing $[g, u]$ is defined by the combinatorial formula

$$
[g, u]\left(x_{0}, \ldots, x_{m+n}\right):=g\left(x_{0}, \ldots, x_{m}\right)\left(u\left(x_{m}, \ldots, x_{m+n}\right)\left(g\left(x_{0}, \ldots, x_{m}\right)^{-1}\right)\right) \text {. }
$$

The pairing (2.11) satisfies the analogue

$$
[g, u]=(-1)^{|g||u|+1}[u, g]
$$

of the graded commutativity rule (2.5), so that its properties may be deduced from those of the pairing $[u, g]$. In particular

$$
\left[g^{-1}, u\right]=-\left[u, g^{-1}\right]=[u, g]^{g} .
$$

We refer to appendix A of [5] for additional properties of these pairings. 


\section{2}

The de Rham differential map

$$
\mathfrak{g} \otimes \mathcal{O}_{S} \Omega_{X / S}^{n} \stackrel{d_{X / S}^{n}}{\longrightarrow} \mathfrak{g} \otimes \mathcal{O}_{S} \Omega_{X / S}^{n+1}
$$

is defined combinatorially for $n \geq 2$, in Alexander-Spanier fashion, by

$$
\mathrm{d}_{X / S}^{n} \omega\left(x_{0}, \ldots, x_{n+1}\right):=\prod_{i=0}^{n+1} \omega\left(x_{0}, \ldots, \widehat{x_{i}}, \ldots \omega_{n+1}\right)^{(-1)^{i}} .
$$

This definition agrees for $n>1$ with the classical definition of the $G$-valued de Rham differential:

$$
\mathrm{d}_{X / S}^{n} \omega:=\mathrm{d}_{X / S} \omega
$$

where for $\omega=Y \otimes \eta$ in $\mathfrak{g} \otimes \Omega_{X / S}^{n}$,

$$
\mathrm{d}_{X / S} \omega:=Y \otimes \mathrm{d} \eta
$$

In particular $\mathrm{d}^{n}$ is an $\mathcal{O}_{S}$-linear map whenever $n \geq 2$, and it follows from (2.15) that the composite $d^{n+1} d^{n}$ is trivial . This also follows from the combinatorial definition of $d^{n}$, since for $n \geq 2$ the factors in the expression (2.13) for $d^{n} \omega$ commute with each other.

For any section $g$ of $G$, we set

$$
\mathrm{d}_{X / S}^{0}(g):=g\left(x_{0}\right)^{-1} g\left(x_{1}\right) .
$$

The map

$$
\begin{gathered}
G_{X} \stackrel{\mathrm{d}_{X / S}^{0}}{\longrightarrow} \mathfrak{g} \otimes \mathcal{O}_{S} \Omega_{X / S}^{1} \\
g \quad \mapsto \quad g^{-1} \mathrm{~d} g
\end{gathered}
$$

is a crossed homomorphism, for the adjoint left action of $G$ on $\mathfrak{g}$. Observe that the expression $g^{-1} \mathrm{~d} g$ is consistent with the combinatorial definition (2.16) of $\mathrm{d}_{X / S}^{0}(g)$. While this traditional expression of $\mathrm{d}_{X / S}^{0}(g)$ as a product of the two terms $g^{-1}$ and $\mathrm{d} g$ does make sense whenever $G$ is a subgroup scheme of the linear group $G L_{n, S}$, such a decomposition is purely conventional for a general $S$-group scheme $G$. A companion to $\mathrm{d}_{X / S}^{0}$ is the differential $\widetilde{\mathrm{d}}^{0}: G \longrightarrow$ $\mathfrak{g} \otimes_{\mathcal{O}_{S}} \Omega_{X / S}^{1}$, defined by

$$
\widetilde{\mathrm{d}}_{X / S}^{0}(g)\left(x_{0}, x_{1}\right):=g\left(x_{1}\right) g\left(x_{0}\right)^{-1} .
$$

The traditional notation for this expression is $d g g^{-1}$. This notation is consistent with such formulas (in additive notation) as 


$$
{ }^{g}\left(g^{-1} d g\right)=\mathrm{d} g g^{-1} \text { and }-\left(g^{-1} \mathrm{~d} g\right)=\mathrm{d} g^{-1} g .
$$

The differential $\mathrm{d}_{X / S}^{1}$ is defined combinatorially by

$$
\left(\mathrm{d}_{X / S}^{1} \omega\right)(x, y, z):=\omega(x, y) \omega(y, z) \omega(z, x) .
$$

In classical terms, it follows (see [4] theorem 3.3) that

$$
\mathrm{d}_{X / S}^{1} \omega:=\mathrm{d} \omega+\frac{1}{2}[\omega, \omega] .
$$

We will henceforth denote $\mathrm{d}_{X / S}^{n}$ simply by $\mathrm{d}^{n}$ for all $n$.

The quadratic term $\frac{1}{2}[\omega, \omega]$ implies that $\mathrm{d}_{X / S}^{1}$ is not a linear map, in fact it follows from (2.19), or the elementary combinatorial calculation of [4] lemma 3.2 , that

$$
\mathrm{d}^{1}\left(\omega+\omega^{\prime}\right)=\mathrm{d}^{1} \omega+\mathrm{d}^{1} \omega^{\prime}+\left[\omega, \omega^{\prime}\right] .
$$

In particular,

$$
\mathrm{d}^{1}(-\omega)=-\mathrm{d}^{1}(\omega)+[\omega, \omega] .
$$

It is immediate, from the combinatorial point of view, that

$$
\mathrm{d}^{1} \mathrm{~d}^{0}(g)=\mathrm{d}^{1}\left(g^{-1} \mathrm{~d} g\right)=0
$$

for all $g$ in $G$. The differential $\mathrm{d}^{1}$ has a companion, which we will denote by $\widetilde{\mathrm{d}}^{1}$, defined by

$$
\widetilde{\mathrm{d}}^{1}(\omega)(x, y, z):=\omega(z, x) \omega(y, z) \omega(x, y) .
$$

A combinatorial computation implies that

$$
\begin{aligned}
\widetilde{\mathrm{d}}^{1} \omega & =\mathrm{d}^{1} \omega-[\omega, \omega] \\
& =\mathrm{d} \omega-\frac{1}{2}[\omega, \omega],
\end{aligned}
$$

and the analogue

$$
\widetilde{\mathrm{d}}^{1}\left(\widetilde{\mathrm{d}}^{0}(g)\right)=\widetilde{\mathrm{d}}^{1}\left(d g g^{-1}\right)=0
$$

of (2.20) is satisfied. Finally, it follows from (2.14) that the $\mathrm{d}^{n}$ satisfy

$$
\mathrm{d}^{i+j}\left[\omega, \omega^{\prime}\right]=\left[\mathrm{d}^{i} \omega, \omega^{\prime}\right]+(-1)^{i}\left[\omega, \mathrm{d}^{j} \omega^{\prime}\right]
$$

whenever $i, j \geq 2$, and the corresponding formula for the pairing $[u, g]$ (2.8) is also valid. 


\section{3}

We now choose, for any $S$-scheme $X$ and any $S$-group scheme $G$, an $\operatorname{Aut}(G)$ valued 1 -form $m$ on $X$. We extend the definition of the de Rham differentials (2.17), (2.18) and (2.12) to the twisted differentials

$$
\mathrm{d}_{X / S, m}^{n}: \mathfrak{g} \otimes_{\mathcal{O}_{S}} \Omega_{X / S}^{n} \longrightarrow \mathfrak{g} \otimes_{\mathcal{O}_{S}} \Omega_{X / S}^{n+1}
$$

(or simply $\mathrm{d}_{m}^{n}$ ) defined combinatorially by the following formulas:

$$
\begin{aligned}
\mathrm{d}_{m}^{1} \omega\left(x_{0}, x_{1}\right): & =\omega\left(x_{0}, x_{1}\right) m\left(x_{0}, x_{1}\right)\left(\omega\left(x_{1}, x_{2}\right)\right) m\left(x_{0}, x_{1}\right) m\left(x_{1}, x_{2}\right)\left(\omega\left(x_{2}, x_{0}\right)\right) \\
& =\omega\left(x_{0}, x_{1}\right) m\left(x_{0}, x_{1}\right)\left(\omega\left(x_{1}, x_{2}\right)\right) \omega\left(x_{0}, x_{2}\right)^{-1} \\
\mathrm{~d}_{m}^{n} \omega\left(x_{0}, \ldots, x_{n+1}\right):= & \\
& =m\left(x_{0}, x_{1}\right)\left(\omega\left(x_{1}, \ldots x_{n+1}\right)\right) \prod_{i=1}^{n+1} \omega\left(x_{0}, \ldots, \widehat{x}_{i}, \ldots, x_{n+1}\right)^{(-1)^{i}}
\end{aligned}
$$

when $n>1$. When the $\operatorname{Aut}(G)$-valued form $m$ is the image $i(\eta)$ under inner conjugation of a $G$-valued form $\eta$, the expression $\mathrm{d}_{i(\eta)}^{n} \omega$ will simply be denoted $\mathrm{d}_{\eta}^{n} \omega$. The corresponding degree zero map $\mathrm{d}_{m}^{0}: G \longrightarrow \mathfrak{g} \otimes_{\mathcal{O}_{S}} \Omega_{X / S}^{1}$ is defined by

$$
\mathrm{d}_{m}^{0}(g):=g\left(x_{0}\right)^{-1} m\left(x_{0}, x_{1}\right)\left(g\left(x_{1}\right)\right)
$$

(and $\mathrm{d}_{m}^{0}(g)$ will also be denoted $g^{-1} \mathrm{~d}_{m}(g)$, consistenly with (2.16) ).

It follows from elementary combinatorial computations that the differentials $\mathrm{d}_{m}^{n}$ can be defined in classical terms by

$$
\mathrm{d}_{m}^{n} \omega=\mathrm{d}^{n} \omega+[m, \omega]
$$

for all $n$, so that for any $\mathfrak{g}$-valued 1 -form $\eta$,

$$
\mathrm{d}_{m+i_{\eta}}^{n}(\omega)=\mathrm{d}_{m}^{n}(\omega)+[\eta, \omega] .
$$

In particular,

$$
\mathrm{d}_{m}^{1}(\omega)=\mathrm{d}^{1} \omega+[m, \omega]=\mathrm{d} \omega+\frac{1}{2}[\omega, \omega]+[m, \omega] .
$$

While the map $\mathrm{d}_{m}^{n}$ is linear for $n \geq 2$,

$$
\mathrm{d}_{m}^{1}\left(\omega+\omega^{\prime}\right)=\mathrm{d}_{m}^{1} \omega+\mathrm{d}_{m}^{1} \omega^{\prime}+\left[\omega, \omega^{\prime}\right]
$$

so that

$$
\mathrm{d}_{m}^{1}(-\omega)=-\mathrm{d}_{m}^{1}(\omega)-[\omega, \omega]
$$


Finally, for any section $g$ of $\Gamma$,

$$
g^{-1} \mathrm{~d}_{m} g=g^{-1} \mathrm{~d} g+[m, g] .
$$

The composite morphism $\mathrm{d}_{m}^{n+1} \mathrm{~d}_{m}^{n}$ is in general non-trivial, and the previous classical definitions of $\mathrm{d}_{m}^{n}$ imply that

$$
\mathrm{d}_{m}^{n+1} \mathrm{~d}_{m}^{n} \omega=\left[\mathrm{d}^{1} m, \omega\right]
$$

whenever $n \geq 2$. For $n=0$, the corresponding formulas are

$$
\mathrm{d}_{m}^{1} \mathrm{~d}_{m}^{0} g=\left[g^{-1}, \mathrm{~d}^{1} m\right] \text { and } \widetilde{\mathrm{d}}_{m}^{1} \widetilde{\mathrm{d}}_{m}^{0} g=\left[\mathrm{d}^{1} m, g\right]
$$

so that, for $n \neq 1$, we recover the well-known assertion that the vanishing of $\mathrm{d}^{1} m=0$ implies that $\mathrm{d}^{n+1} \mathrm{~d}^{n}=0$. One verifies that for any 1 -form $\omega$

$$
\begin{aligned}
\mathrm{d}_{m}^{2} \mathrm{~d}_{m}^{1}(\omega) & =\left[\mathrm{d}^{1} m, \omega\right]+\left[\mathrm{d}_{m}^{1} \omega, \omega\right] \\
& =\left[\mathrm{d}^{1} m, \omega\right]+\left[\mathrm{d}^{1} \omega, \omega\right]+[[m, \omega], \omega] .
\end{aligned}
$$

This reduces to the equation

$$
\mathrm{d}_{m}^{2} \mathrm{~d}_{m}^{1}(\omega)=\left[\mathrm{d}^{1} m, \omega\right]
$$

of type (2.26) whenever $\mathrm{d}_{m}^{1} \omega=0$. For $m=i(\omega)$, equation (2.28) is equivalent to the classical Bianchi identity [9] II Theorem 5.4:

$$
\mathrm{d}_{\omega}^{2} \mathrm{~d}^{1} \omega=0 \text {. }
$$

We now state the functoriality properties of the differential (2.22) $\mathrm{d}_{m}^{n}$ for $n \geq 1$. We define the twisted conjugate ${ }^{g *} \omega$ of a $G$-valued 1 -form $\omega$ by

$$
\begin{aligned}
g * \omega:=\left(p_{0}^{*} g\right) \omega\left(p_{1}^{*} g\right)^{-1} & ={ }^{g} \omega+g \mathrm{~d} g^{-1} \\
& =\omega+[g, \omega]+g \mathrm{~d} g^{-1} .
\end{aligned}
$$

It follows from the combinatorial definition (2.18) of $\mathrm{d}^{1}$ that

$$
{ }^{g}\left(\mathrm{~d}^{1} \omega\right)=\mathrm{d}^{1}\left({ }^{g *} \omega\right) .
$$

More generally, for any $G$-valued form $\omega$ of degree $n \geq 1$, and any section $u$ of $\operatorname{Aut}(G)$ on $X$,

$$
\begin{aligned}
u\left(\mathrm{~d}_{m}^{n}(\omega)\right) & =\mathrm{d}_{\left({ }^{u *} m\right)}^{n} u(\omega) \\
& =\mathrm{d}_{\left({ }^{u} m\right)}^{n} u(\omega)+\left[u \mathrm{~d} u^{-1}, u(\omega)\right] \\
& =\mathrm{d}_{m}^{n}(u(\omega))+[[u, m], u(\omega)]+\left[u \mathrm{~d} u^{-1}, u(\omega)\right]
\end{aligned}
$$




\section{Gerbes and their connective structures}

\section{1}

Let $\mathcal{P}$ be a gerbe 3 on an $S$-scheme $X$. For simplicity, in discussing gerbes we will make two additional assumptions:

- $\mathcal{P}$ is a $G$-gerbe, for a given $S$-group scheme $G$.

- $\mathcal{P}$ is connected.

The first assumption gives us, for any object $x$ in the fibre category $\mathcal{P}_{U}$ above an open set $U \subset X$, an isomorphism of sheaves on $U$

$$
G_{\mid U} \stackrel{\sim}{\longrightarrow} \operatorname{Aut}_{\mathcal{P}_{U}}(x)
$$

The second assumption asserts that for any pair of objects $x, y \in \operatorname{ob}\left(\mathcal{P}_{U}\right)$ there exists an arrow $x \longrightarrow y$ in the category $\mathcal{P}_{U}$. This ensures that the gerbe is described by an element in the degree 2 Cech cohomology of $X$ rather than by degree 2 cohomology with respect to a hypercover of $X$.

Let us choose a family of local objects $x_{i} \in \mathcal{P}_{U_{i}}$, for some open cover $\mathcal{U}=\coprod_{i} U_{i}$ of $X$, and a family of arrows

$$
x_{j} \stackrel{\phi_{i j}}{\longrightarrow} x_{i}
$$

in $\mathcal{P}_{U_{i j}}$. Identifying elements of both $\operatorname{Aut}_{\mathcal{P}}\left(x_{i}\right)$ and $\operatorname{Aut}_{\mathcal{P}}\left(x_{j}\right)$ with the corresponding sections of $G$ above $U_{i}$ and $U_{j}$, these arrows determine a family of section $\lambda_{i j} \in \Gamma\left(U_{i j}\right.$, Aut $\left.(G)\right)$, defined by the commutativity of the diagrams

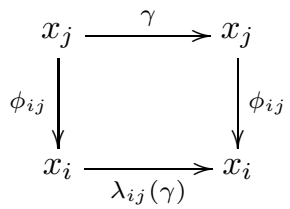

for every $\gamma \in G_{\mid U_{i j}}$. In addition, the arrows $\phi_{i j}$ determine a family of elements $g_{i j k} \in G_{\mid U_{i j k}}$ for all $(i, j, k)$ by the commutativity of the diagrams

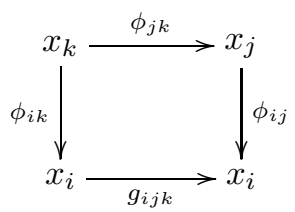

\footnotetext{
${ }^{3}$ We refer to 3 and 6 for the definition of a gerbe, and for additional details regarding the associated cocycle and coboundary equations (3.7), (3.14).
} 
above $U_{i j k}$. By conjugation in the sense made clear by diagram (3.3), it follows that the $\lambda_{i j}$ satisfy the cocycle condition

$$
\lambda_{i j} \lambda_{j k}=i\left(g_{i j k}\right) \lambda_{i k} .
$$

By [6] lemma 5.1, the $G$-valued cochains $g_{i j k}$ also satisfy the cocycle condition

$$
\lambda_{i j}\left(g_{j k l}\right) g_{i j l}=g_{i j k} g_{i k l} .
$$

These two cocycle equations may be written more compactly as

$$
\begin{cases}\delta^{1} \lambda_{i j} & =i\left(g_{i j k}\right) \\ \delta_{\lambda_{i j}}^{2}\left(g_{i j k}\right) & =1\end{cases}
$$

where $\delta_{\lambda}^{2}$ is the $\lambda$-twisted degree 2 Čech differential determined by equation (3.6). They may be jointly viewed as the $(G \longrightarrow \operatorname{Aut}(G))$-valued Čech 1cocycl 4 equations associated to the gerbe $\mathcal{P}$, the open cover $\mathcal{U}$ of $X$, and the trivializing families of objects $x_{i}$ and arrows $\phi_{i j}$ in $\mathcal{P}$.

Let us choose a second family of local objects $x_{i}^{\prime}$ in $\mathcal{P}_{U_{i}}$, and of arrows

$$
x_{j}^{\prime} \stackrel{\phi_{i j}^{\prime}}{\longrightarrow} x_{i}^{\prime}
$$

above $U_{i j}$. To these correspond a new cocycle pair $\left(\lambda_{i j}^{\prime}, g_{i j k}^{\prime}\right)$. In order to compare this set of arrows with the previous one, we choose (after a harmless refinement of the given open cover $\mathcal{U}$ of $X$ ) a family of arrows

$$
x_{i} \stackrel{\chi_{i}}{\longrightarrow} x_{i}^{\prime}
$$

in $\mathcal{P}_{U_{i}}$ for all $i$. The arrow $\chi_{i}$ induces by conjugation a section $r_{i}$ in the group of sections $\Gamma\left(U_{i}, \operatorname{Aut}(G)\right)$, characterized by the commutativity of the square

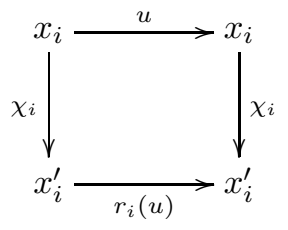

for all $u \in G$. The lack of compatibility between these arrows $\chi_{i}$ and the arrows $\phi_{i j}, \phi_{i j}^{\prime}$ (3.2), (3.8) is measured by the family of sections $\vartheta_{i j} \in \Gamma\left(U_{i j}, G\right)$ determined by the commutativity of the following diagram:

\footnotetext{
${ }^{4}$ We prefer to emphasize the fact that $\lambda_{i j}$ is a 1-cochain since this is more consistent with a simplicial definition of the associated cohomology, even though it is more customary to view the pair of equations (3.7) as a 2-cocycle equation, with (3.5) an auxiliary condition.
} 


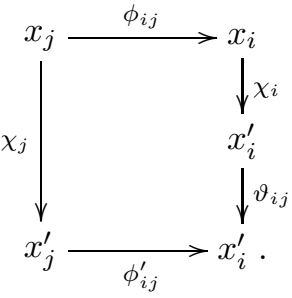

Under the identifications (3.1), diagram (3.11) induces by conjugation, in a sense made clear by the definition (3.10) of the auromorphism $r_{i}$, a commutative diagram of group schemes above $U_{i j}$

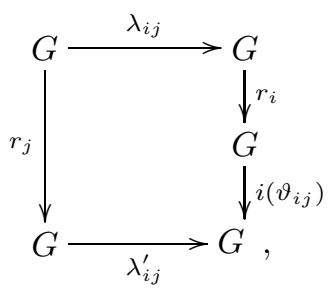

whose commutativity is expressed by the equation

$$
\lambda_{i j}^{\prime}=i\left(\vartheta_{i j}\right) r_{i} \lambda_{i j} r_{j}^{-1}
$$

in $\operatorname{Aut}(G)$.

Consider now the diagran 5

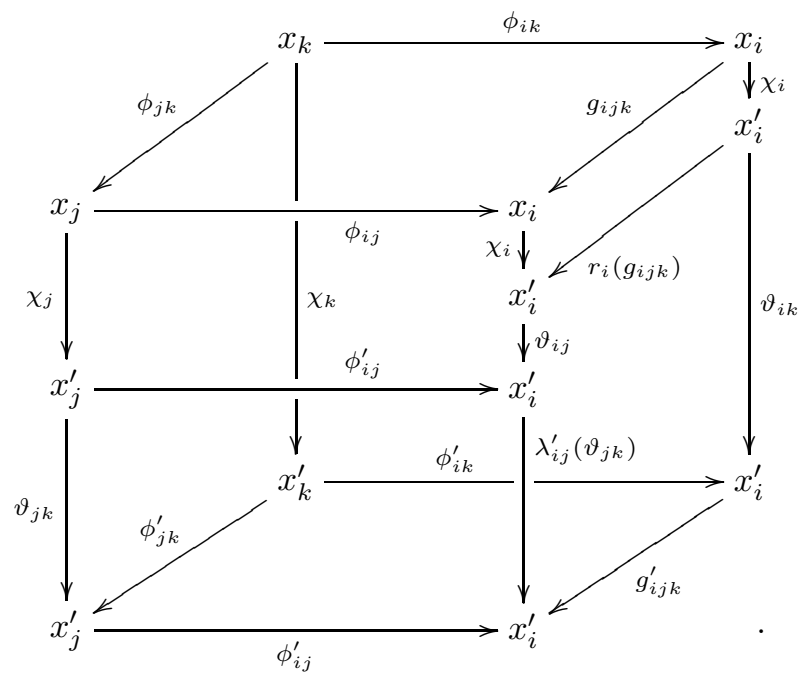

\footnotetext{
${ }^{5}$ This diagram whose faces are five pentagons and three squares (as well as those in (4.9) and (4.25) below) is the 1-skeleton of a Saneblidze-Umble cubical model [15], [13] for the Stasheff associahedron $K_{5}$ [17].
} 
Both the top and the bottom squares commute, since these squares are of type (3.4). So do the back, the left and the top front vertical squares, since all three are of type (3.11). The same is true of the lower front square, and the upper right vertical square, since these two are respectively of the form (3.3) and (3.10). It follows that the remaining lower right square in the diagram is also commutative, since all the arrows in diagram (3.13) are invertible. The commutativity of this final square is expressed algebraically by the equation

$$
g_{i j k}^{\prime} \vartheta_{i k}=\lambda_{i j}^{\prime}\left(\vartheta_{j k}\right) \vartheta_{i j} r_{i}\left(g_{i j k}\right) .
$$

We say that two cocycle pairs $\left(\lambda_{i j}, g_{i j k}\right)$ and $\left(\lambda_{i j}^{\prime}, g_{i j k}^{\prime}\right)$ are cohomologous if we are given a pair $\left(r_{i}, \vartheta_{i j}\right)$, with $r_{i} \in \Gamma\left(U_{i}, \operatorname{Aut}(G)\right)$ and $\vartheta_{i j} \in \Gamma\left(U_{i j}, G\right)$, satisfying those two equations

$$
\begin{cases}\lambda_{i j}^{\prime} & =i\left(\vartheta_{i j}\right) r_{i} \lambda_{i j} r_{j}^{-1} \\ g_{i j k}^{\prime} \vartheta_{i k} & =\lambda_{i j}^{\prime}\left(\vartheta_{j k}\right) \vartheta_{i j} r_{i}\left(g_{i j k}\right) .\end{cases}
$$

and display this as

$$
\left(\lambda_{i j}, g_{i j k}\right) \stackrel{\left(r_{i}, \vartheta_{i j}\right)}{\sim}\left(\lambda_{i j}^{\prime}, g_{i j k}^{\prime}\right)
$$

The equivalence class of the cocycle pair $\left(\lambda_{i j}, g_{i j k}\right)$ for this relation is independent of the choices of objects $x_{i}$ and arrows $\phi_{i j}$ by from which it was constructed. By definition, it determines an element in the first non-abelian Čech cohomology set $\check{H}^{1}(\mathcal{U}, G \stackrel{i}{\longrightarrow} \operatorname{Aut}(G))$ with coefficients in the crossed module $i: G \longrightarrow \operatorname{Aut}(G)$.

\section{2}

In [5], the combinatorial description of differential forms is used in order to define the concepts of connections and curvings on a gerbe. For any $S$-group scheme $G$, a (relative) connection on a principal $G$-bundle $P$ above the $S$ scheme $X$ may be defined as a morphism

$$
p_{1}^{*} P \stackrel{\epsilon}{\longrightarrow} p_{0}^{*} P
$$

between the two pullbacks of $P$ to $\Delta_{X / S}^{1}$, whose restriction to the diagonal subscheme

$$
\Delta: X \hookrightarrow \Delta_{X / S}^{1}
$$

is the identity morphism $1_{P}$.

This type of definition of a connection, as a vehicle for parallel transport, remains valid for other structures than principal bundles. In particular, for any $X$-group scheme $\Gamma$, a connection on $\Gamma$ is a morphism of group schemes 


$$
\mu: p_{1}^{*} \Gamma \longrightarrow p_{0}^{*} \Gamma
$$

above $\Delta_{X / S}^{1}$ whose restriction to the diagonal subscheme $X \hookrightarrow \Delta_{X / S}^{1}$ is the identity morphism $1_{\Gamma}$. When $\Gamma$ is the pullback to $X$ of an $S$-group scheme $G$, the inverse images $p_{1}^{*} G$ and $p_{0}^{*} G$ of $G_{X}$ above $\Delta_{X / S}^{1}$ are canonically isomorphic, so that the connection (3.17) is then described by a $\operatorname{Lie}(\operatorname{Aut}(G))$-valued 1-form $m$.

A connection $\mu$ on a group $\Gamma$ determines de Rham differentials

$$
\mathrm{d}_{X / S, \mu}^{n}: \operatorname{Lie}(\Gamma) \otimes_{\mathcal{O}_{S}} \Omega_{X / S}^{n} \longrightarrow \operatorname{Lie}(\Gamma) \otimes_{\mathcal{O}_{S}} \Omega_{X / S}^{n+1}
$$

(or simply $\mathrm{d}_{\mu}^{n}$ ) defined combinatorially by the formulas [5] (A.1.9)-(A.1.11) and their higher analogues. When $\Gamma$ is the pullback of an $S$-group scheme, $\mathrm{d}_{\mu}^{n}$ is decribed in classical terms as the deformation (2.22)

$$
\mathrm{d}_{\mu}^{n}:=\mathrm{d}_{m}^{n}
$$

of the de Rham differential $\mathrm{d}^{n}$ determined by the associated 1-form $m$. When the curvature $\mathrm{d}^{1} m$ of the connection $\mu$ is trivial, the connection is said to be integrable. In that case, it follows from (2.26) and (2.27) that the de Rham differentials satisfy the condition $\mathrm{d}_{m}^{n+1} \mathrm{~d}_{m}^{n}=0$ for all $n \neq 1$.

The curvature of a connection $\epsilon$ (3.16) on a principal bundle $P$ is the unique arrow

$$
\kappa_{\epsilon}: p_{0}^{*} P \longrightarrow p_{0}^{*} P
$$

such that the following diagram above $\Delta_{X / S}^{2}$ commutes, with $\epsilon_{i j}$ the pullbacks of $\epsilon$ under the corresponding projections $p_{i j}: \Delta_{X / S}^{2} \longrightarrow \Delta_{X / S}^{1}$ :

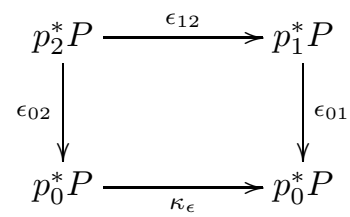

By construction, $\kappa_{\epsilon}$ is a relative 2 -form on $X$ with values in the gauge group $P^{\text {ad }}:=\operatorname{Isom}_{G}(P, P)$ of $P$.

The connection $\epsilon$ on $P$ induces a connection $\mu_{\epsilon}$ on the group $P^{\text {ad }}$, determined by the commutativity of the squares

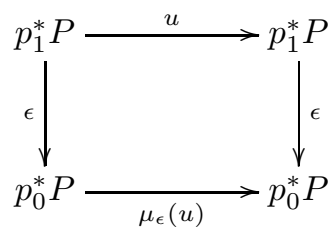


for all sections $u$ of $p_{1}^{*}\left(P^{\mathrm{ad}}\right)$. By [1], [5] proposition 1.7, the curvature 2-form $\kappa_{\epsilon}$ satisfies the Bianchi identity

$$
\mathrm{d}_{\mu_{\epsilon}}^{2}\left(\kappa_{\epsilon}\right)=0
$$

For a given family of local sections of $P$, with associated $G$-valued 1-cocycles $g_{i j}$, the connection (3.16) is described by a family of $G$-valued 1-forms $\omega_{i} \in$ $\mathfrak{g} \otimes \Omega_{U_{i} / S}^{1}$, satisfying the gluing condition

$$
\omega_{j}=\omega_{i}^{* g_{i j}}=\omega_{i}^{g_{i j}}+g_{i j}^{-1} \mathrm{~d} g_{i j}
$$

above $U_{i j}$, for the action of $G$ on $\mathfrak{g} \otimes_{\mathcal{O}_{S}} \Omega_{U_{i} / S}^{1}$ induced by the adjoint right action of $G$ on $\mathfrak{g}$. A 1-form satisfying this equation is classically known as a connection form. The induced curvature $\kappa$ is locally described by the family of 2 -forms

$$
\kappa_{i}:=\mathrm{d}^{1} \omega_{i}=\mathrm{d} \omega_{i}+\frac{1}{2}\left[\omega_{i}, \omega_{i}\right],
$$

and these satisfy the simpler Čech (or gluing) condition

$$
\kappa_{j}=\kappa_{i}^{g_{i j}} .
$$

Equation (3.19) is reflected at the local level in the equation

$$
\mathrm{d}_{\omega_{i}}^{2} \kappa_{i}=0,
$$

which is simply the classical Bianchi identity (2.30) for the 1-form $\omega_{i}$.

\section{3}

The notion of a connective structure on a $G$-gerbe $\mathcal{P}$ is a categorification of the notion of a connection on a principal bundle, as we will now recall, following [5] $\S 4$. To $\mathcal{P}$ is associated its gauge stack $\mathcal{P}^{\text {ad }}$. By definition this is the monoidal stack $\mathcal{E}_{q_{X}}(\mathcal{P}, \mathcal{P})$ of self-equivalences of the stack $\mathcal{P}$, the monoidal structure being defined by the composition of equivalences. A connection on a $\mathcal{P}$ is an equivalence between stacks

$$
p_{1}^{*} \mathcal{P} \stackrel{\epsilon}{\longrightarrow} p_{0}^{*} \mathcal{P}
$$

above $\Delta_{X / S}^{1}$, together with a natural isomorphism between the restriction $\Delta^{*} \epsilon$ of $\epsilon$ to the diagonal subscheme $X$ of $\Delta_{X / S}^{1}$ and the identity morphism $1_{\mathcal{P}}$. Such a connection $\epsilon$ induces as in (3.18) a connection $\mu$ on the gauge stack $\mathcal{P}^{\text {ad }}$. 
A curving of $(\mathcal{P}, \epsilon)$ is a natural isomorphism $K$

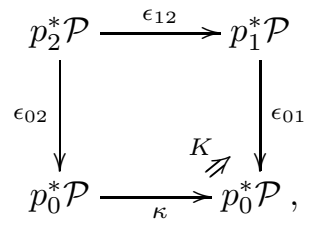

for some morphism

$$
\kappa: p_{0}^{*} \mathcal{P} \longrightarrow p_{0}^{*} \mathcal{P}
$$

above $\Delta_{X / S}^{2}$. It is determined by the choice of some explicit quasi-inverse of the connection $\epsilon$. The arrow $\kappa$ which arises as part of the definition of $K$ is called the fake curvature associated to the connective structure $(\epsilon, K)$. It is a global object in the pullback to $\Delta_{X / S}^{2}$ of the gauge stack $\mathcal{P}^{\text {ad }}$.

The connective structure $(\epsilon, K)$ determines a 2-arrow

$$
\begin{aligned}
p_{0}^{*} \mathcal{P} \stackrel{\kappa_{013}}{\longrightarrow} p_{0}^{*} \mathcal{P} \\
\left.p_{0}^{*} \mathcal{P} \stackrel{\kappa_{012}}{\longleftarrow}\right|_{\kappa_{012}} ^{\longrightarrow} \mathcal{P}
\end{aligned}
$$

This is the unique 2-arrow which may be inserted in diagram

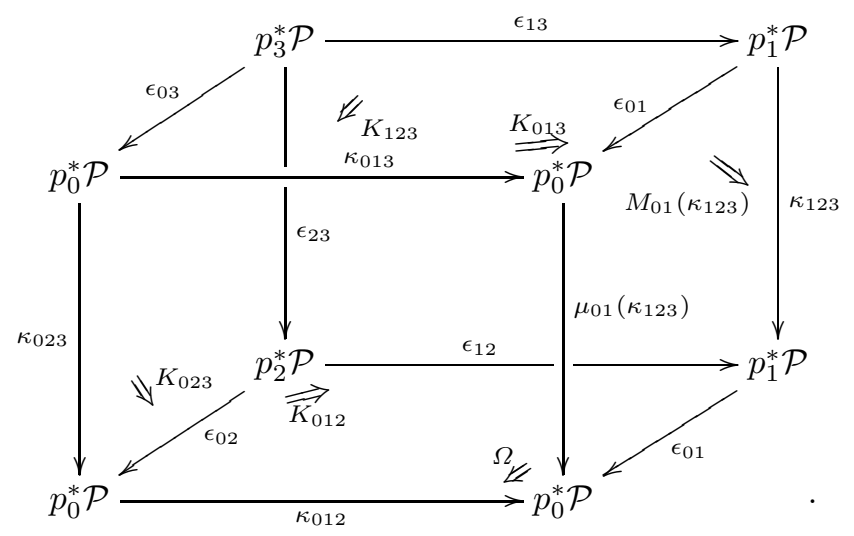

so that the two composite 2-arrows

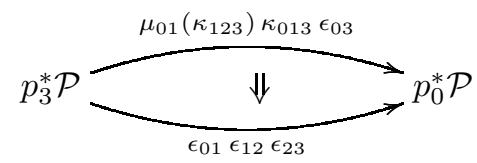

which may be constructed by composition of 2-arrows in (3.23) coincide. 
This 2-arrow $\Omega$ may also be viewed as a 1-arrow above $\Delta_{X / S}^{3}$ in the gauge group $\mathcal{P}^{\text {ad }}$, or even as an arrow in the stack $\operatorname{Lie}\left(\mathcal{P}^{\text {ad }}\right) \otimes_{\mathcal{O}_{S}} \Omega_{X / S}^{3}$ of relative $\operatorname{Lie}\left(\mathcal{P}^{\text {ad }}\right)$-valued 3 -forms on $X$. Returning to the combinatorial definition [5] (A.1.10) of the de Rham differential, we may finally view $\Omega$, by horizontal composition with appropriate 1 -arrows, as a 1 -arrow in $\mathcal{P}^{\text {ad }}$ whose source object is the identity arrow $I_{\mathcal{P} \text { ad }}$ :

$$
I \stackrel{\Omega}{\longrightarrow} \mathrm{d}_{\mu}^{2}\left(\kappa^{-1}\right)
$$

Denoting the twisted differential $\mathrm{d}_{\mu}^{2}$ by the expression $\mathrm{d}+[\mu$,$] to which it$ reduces when appropriate trivializations have been chosen, the 3 -curvature arrow $\Omega(3.24)$ is described by the equation (1.4). By [5] theorem 4.4 it satisfies another relation, described by the cubical pasting diagram [5] (4.1.24), and which may be expressed by the higher Bianchi identity 6 (1.5). The pair of equations (1.4) and (1.5) may now be thought of as a categorified version, satisfied by the pair of $\mathcal{P}^{\text {ad }}$-valued forms $(\kappa, \Omega)$, of the classical Bianchi identity (3.19), and can be written in symbolic form as

$$
\mathrm{d}_{\mu, \mathcal{K}}^{2}(\kappa, \Omega)=0,
$$

where $\mathrm{d}_{\mu, \mathcal{K}}^{n}$ is the twisted de Rham differential on $\operatorname{Lie}\left(\mathcal{P}^{\text {ad }}\right)$-valued $n$-forms determined by twisting data $(\mu, \mathcal{K})$ associated to the given connective structure on $\mathcal{P}$.

\section{4 Čech-de Rham cocycles}

\section{1}

We observed in section 3, 1 that a gerbe could be expressed in cocyclic terms, once local trivializations were chosen. We will now show that this is also the case for the connection $\epsilon$. We choose, for each $i \in I$, an arrow

$$
\gamma_{i}: \epsilon p_{1}^{*} x_{i} \longrightarrow p_{0}^{*} x_{i}
$$

in $p_{0}^{*} \mathcal{P}_{U_{i}}$ such that $\Delta^{*} \gamma_{i}=1_{x_{i}}$. The arrow $\gamma_{i}$ determines by conjugation a connection

$$
m_{i}: p_{1}^{*} G_{\mid U_{i}} \longrightarrow p_{0}^{*} G_{\mid U_{i}}
$$

on the pullback $G_{\mid U_{i}}$ of the group $G$ above the open set $U_{i} \subset X$. The arrow $m_{i}$ is described, for any section $g \in \Gamma\left(\Delta_{X / S_{U_{i}}}^{1}, p_{1}^{*} G\right)$, by the commutativity of the diagram

\footnotetext{
${ }^{6}$ See [5] (4.1.28) for a proof of this identity.
} 


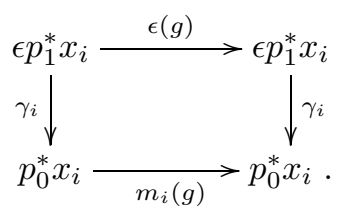

The pair $\left(\phi_{i j}, \gamma_{i}\right)$ determines a family of arrows $\gamma_{i j}$ in the pullback $G_{\Delta_{U_{i j}}^{1}}$ of $G$, defined by the commutativity of the diagram

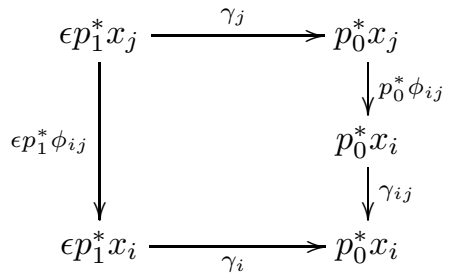

By conjugation, this determines a commutative diagram

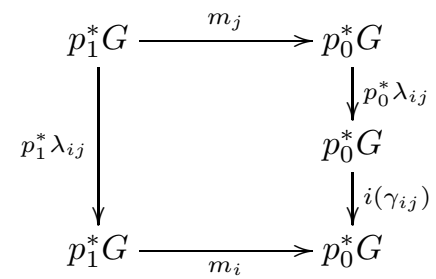

so that the equation

$$
i\left(\gamma_{i j}\right)\left(p_{0}^{*} \lambda_{i j}\right) m_{j}\left(p_{1}^{*} \lambda_{i j}\right)^{-1}=m_{i} .
$$

of [5] (6.1.2) is satisfied.

We may restate (4.5) as

$$
i\left(\gamma_{i j}\right)\left[\left(p_{0}^{*} \lambda_{i j}\right) m_{j}\left(p_{0}^{*} \lambda_{i j}\right)^{-1}\right]=m_{i}\left[p_{1}^{*} \lambda_{i j}\left(p_{0}^{*} \lambda_{i j}^{-1}\right)\right],
$$

an equation all of whose factors are $\operatorname{Aut}(G)$-valued 1-forms on $U_{i j}$ and therefore commute with each other. In the notation introduced in (2.31), equation (4.6) can be rewritten as

$$
\lambda_{i j}^{*} m_{j}=m_{i}-i\left(\gamma_{i j}\right),
$$

or more classically as

$$
{ }^{\lambda_{i j}} m_{j}=m_{i}-\lambda_{i j} \mathrm{~d} \lambda_{i j}^{-1}-i\left(\gamma_{i j}\right) .
$$

This is is the analogue for the Aut $(G)$-valued forms $m_{i}$ and $\lambda_{i j}$ of the classical expression (3.20) for a connection form, but now categorified by the insertion of an additional summand $-i\left(\gamma_{i j}\right)$. 
Consider now the following diagr in $\mathcal{P}_{\Delta_{U_{i j k}}^{1}}$ :

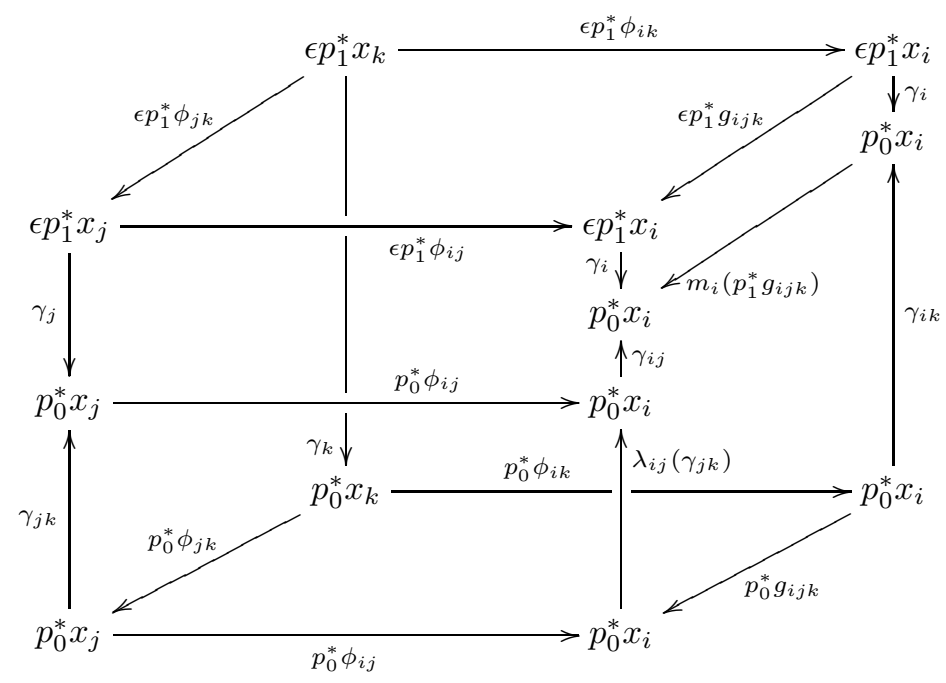

Of the eight faces of this cube, seven are known to be commutative. It follows that the remaining lower square on the right vertical side is also commutative. This is the square

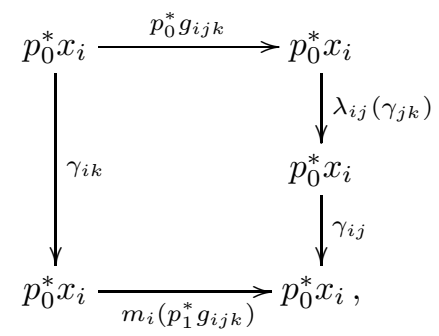

whose commutativity corresponds to the equation

$$
\gamma_{i j}\left(p_{0}^{*} \lambda_{i j}\left(\gamma_{j k}\right)\right)=m_{i}\left(p_{1}^{*} g_{i j k}\right) \gamma_{i k}\left(p_{0}^{*} g_{i j k}\right)^{-1}
$$

in other words to the equation [5] (6.1.7), all of whose factors are $G$-valued 1 -forms on $U_{i j k}$. We may rewrite this as

$$
\gamma_{i j} p_{0}^{*} \lambda_{i j}\left(\gamma_{j k}\right)=\left(m_{i}\left(p_{1}^{*} g_{i j k}\right) p_{0}^{*} g_{i j k}^{-1}\right)\left(p_{0}^{*} g_{i j k} \gamma_{i k} p_{0}^{*} g_{i j k}^{-1}\right)
$$

so that, taking into account the equation (3.5), we finally obtain (in additive notation)

$$
\gamma_{i j}+\lambda_{i j}\left(\gamma_{j k}\right)-\lambda_{i j} \lambda_{j k}\left(\lambda_{i k}^{-1}\left(\gamma_{i k}\right)\right)=d g_{i j k} g_{i j k}^{-1}+\left[m_{i}, g_{i j k}\right],
$$

with bracket defined by (2.7) an equation which can be written in abbreviated form as

$$
\delta_{\lambda_{i j}}^{1}\left(\gamma_{i j}\right)=\mathrm{d}_{m_{i}} g_{i j k} g_{i j k}^{-1} .
$$




\section{2}

We now describe in similar terms the curving $K$ and the fake curvature $\kappa$ of diagram (3.22). Just as we associated to the connection $\epsilon$ (3.21) a family of arrows $\gamma_{i}$ (4.1), we now choose, for each $i \in I$, an arrow

$$
\kappa p_{0}^{*} x_{i} \stackrel{\delta_{i}}{\longrightarrow} p_{0}^{*} x_{i}
$$

in the category $\mathcal{P}_{\Delta_{U_{i}}^{2}}$, whose restriction to the degenerate subsimplex $s \Delta_{U_{i}}^{2}$ of $\Delta_{U_{i}}^{2}$ is the identity. To the curving $K$ is associated a family of " $B$-field" $\mathfrak{g}$-valued 2-forms $B_{i} \in \mathfrak{g} \otimes \Omega_{U_{i}}^{2}$, characterized by the commutativity of the following diagram 7 in which an expression such as $\gamma_{i}^{12}$ is the pullback of $\gamma_{i}$ by the corresponding projection $p_{12}: \Delta_{X / S}^{2} \longrightarrow \Delta_{X / S}^{1}$ :

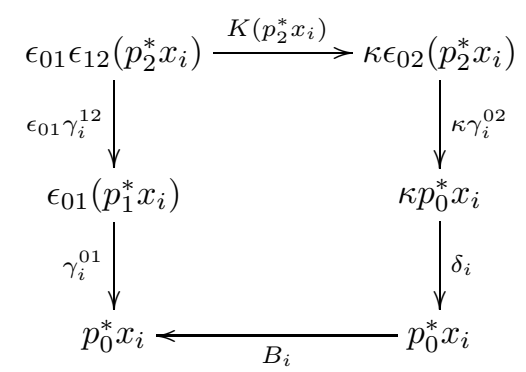

Let us now define a family of $G$-valued 2 -forms $\nu_{i}$ on $U_{i}$ by the equations

$$
\nu_{i}:=\mathrm{d}^{1} m_{i}-i\left(B_{i}\right)
$$

in Lie $\operatorname{Aut}(G) \otimes \Omega_{U_{i}}^{2}$, in other words by the commutativity of the diagram

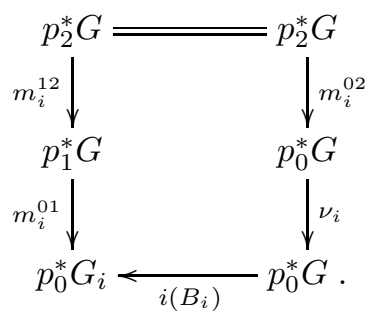

By comparing diagram (4.15) with the conjugate of diagram (4.13), we see that $\nu_{i}$ is simply the conjugate of the arrow $\delta_{i}$. It can therefore described by the commutativity of the diagram

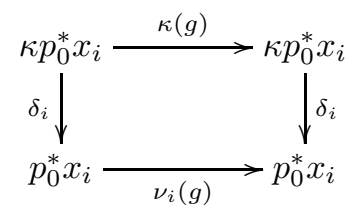

\footnotetext{
${ }^{7}$ The chosen orientation of the arrow $B_{i}$ is consistent with that in [5].
} 
for all $g \in \Gamma\left(\Delta_{U_{i} / S}^{2}, p_{0}^{*} G\right)$, just as the connection $m_{i}$ was described by diagram (4.2).

We also define a family of 2 -forms $\delta_{i j}$ by the commutativity of the diagram

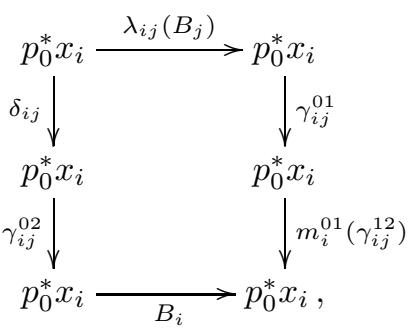

i.e., since all terms commute, by the equation

$$
\delta_{i j}:=\lambda_{i j}\left(B_{j}\right)-B_{i}-\mathrm{d}_{m_{i}}^{1}\left(-\gamma_{i j}\right)
$$

in $\operatorname{Lie}(G) \otimes \Omega_{U_{i} / S}^{2}$. In Čech-de Rham notation, this is

$$
\delta_{i j}:=\delta_{\lambda_{i j}}^{0}\left(B_{i}\right)-\mathrm{d}_{m_{i}}^{1}\left(-\gamma_{i j}\right),
$$

and in classical notation

$$
\delta_{i j}:=\lambda_{i j}\left(B_{j}\right)-B_{i}+\mathrm{d} \gamma_{i j}-\frac{1}{2}\left[\gamma_{i j}, \gamma_{i j}\right]+\left[m_{i}, \gamma_{i j}\right] .
$$

Here is another characterization of $\delta_{i j}$ :

Lemma 4.1. For every pair $(i, j) \in I$, the analogue

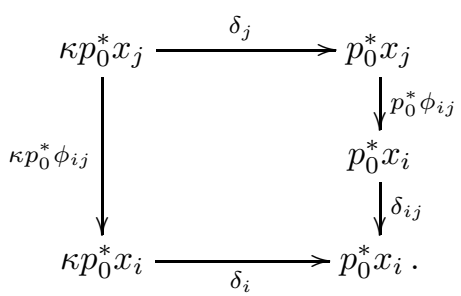

of diagram (4.3) is commutative. 
Proof: Consider the diagram

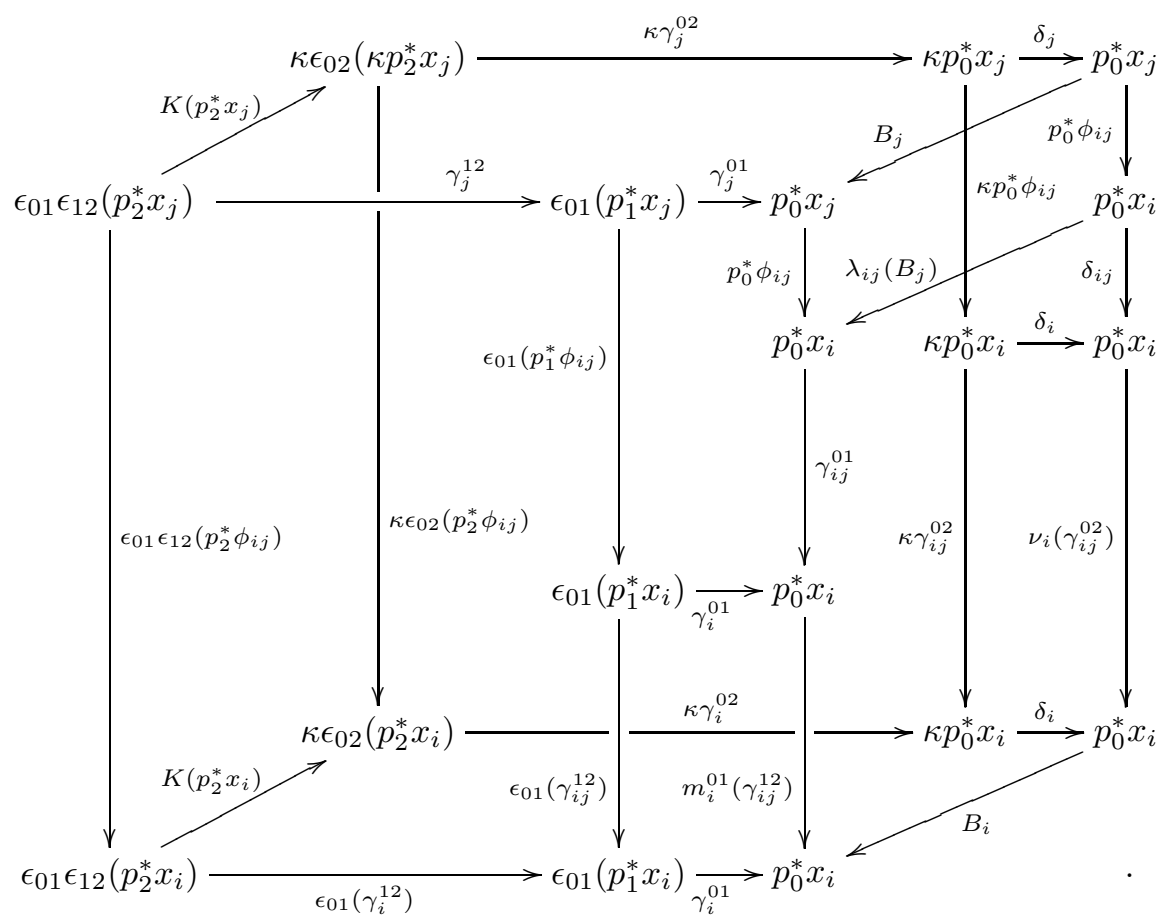

Diagrams (4.13), (4.17) and (4.16) imply that all squares in (4.20) are commutativ 8 , except possibly the rear right upper one. This remaining square (4.19) is therefore also commutative.

Conjugating diagram (4.19), we obtain as in (4.4) a square

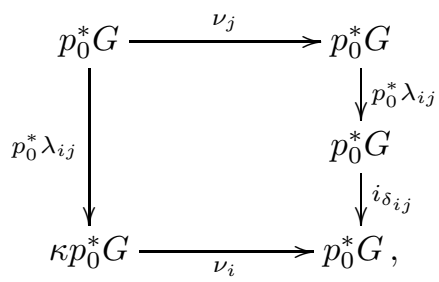

whose commutativity is expressed algebraically as

$$
i\left(\delta_{i j}\right)\left(p_{0}^{*} \lambda_{i j}\right) \nu_{j}=\nu_{i}\left(p_{0}^{*} \lambda_{i j}\right) .
$$

${ }^{8}$ This is true for diagram (4.17) since $\nu_{i}\left(\gamma_{i j}^{02}\right)=\gamma_{i j}^{02}$. 
In additive notation, this is equation

$$
{ }^{\lambda_{i j}} \nu_{j}=\nu_{i}-i\left(\delta_{i j}\right),
$$

in other words

$$
\delta_{\lambda_{i j}}^{0} \nu_{i}=-i\left(\delta_{i j}\right)
$$

It is instructive to note that this equation can be derived directly from equation (4.8) and the definitions (4.14) and (4.18) of $\nu_{i}$ and $\delta_{i j}$. First of all, observe that by (2.32)

$$
\mathrm{d}^{1}\left({ }^{\lambda_{i j} *} m_{i}\right)={ }^{\lambda_{i j}}\left(\mathrm{~d}^{1} m_{i}\right)
$$

One then computes

$$
\begin{aligned}
{ }^{\lambda_{i j}} \nu_{j} & ={ }^{\lambda_{i j}}\left(\mathrm{~d}^{1}\left(m_{j}\right)-i_{B_{j}}\right) \\
& =\mathrm{d}^{1}\left({ }^{\lambda_{i j} *} m_{j}\right)-i\left(\lambda_{i j}\left(B_{j}\right)\right) \\
& =\mathrm{d}^{1}\left(m_{i}-i\left(\gamma_{i j}\right)\right)-i\left(B_{i}+\mathrm{d}_{m_{i}}^{1}\left(-\gamma_{i j}\right)+\delta_{i j}\right) \\
& =\mathrm{d}^{1} m_{i}-\mathrm{d}^{1}\left(i\left(\gamma_{i j}\right)\right)-\left[m_{i}, \gamma_{i j}\right]-i\left(B_{i}\right)-i\left(\mathrm{~d}^{1} m_{i}\left(-\gamma_{i j}\right)\right)-i\left(\delta_{i j}\right) .
\end{aligned}
$$

Since the homomorphism $i$ commutes with $\mathrm{d}^{1} m$ and $\left[m_{i}, i\left(\gamma_{i j}\right)\right]=i\left(\left[m_{i}, \gamma_{i j}\right]\right)$, the summands $i\left(\mathrm{~d}^{1} m\left(-\gamma_{i j}\right)\right)$ and $\mathrm{d}^{1}\left(i\left(\gamma_{i j}\right)\right)+\left[m_{i}, \gamma_{i j}\right]$ cancel out. The first two remaining summands describe $\nu_{i}$, so that equation (4.22) is satisfied.

In the same vein, the analogue for the fake curvature $\kappa$ of (4.10) is the following assertion.

Lemma 4.2. The diagram

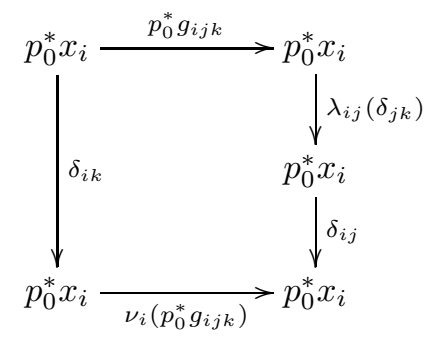

is commutative. 
Proof: By (4.19), (3.4) and (4.16), all squares in the diagram

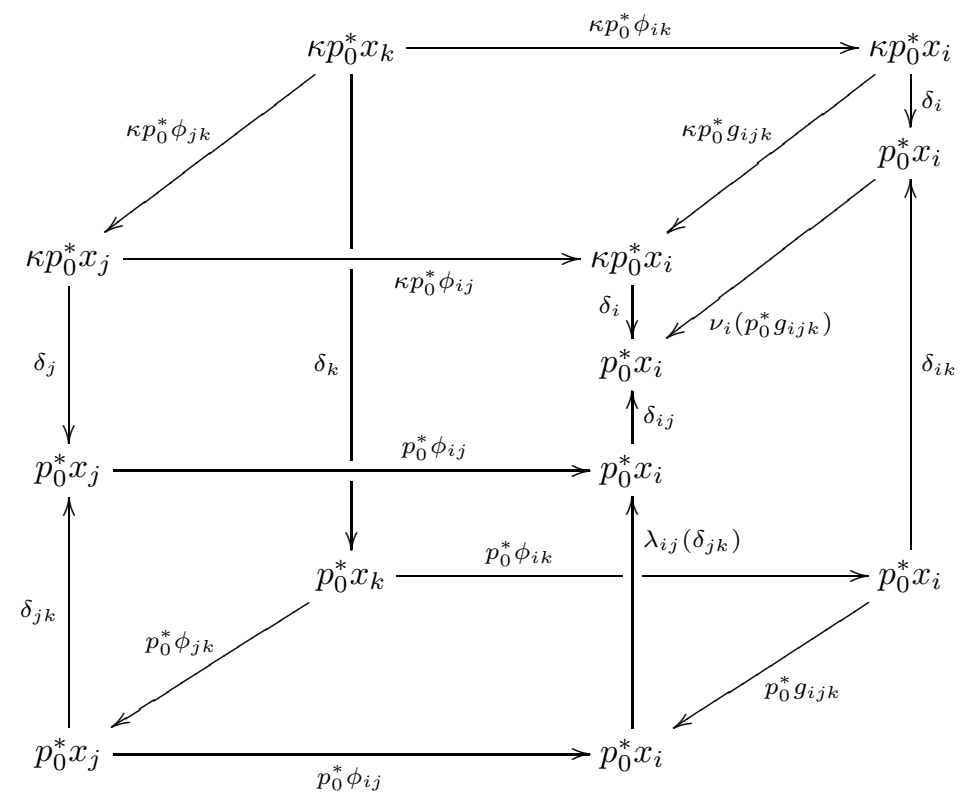

are commutative, except possibly the lower right-hand one. It follows that the latter one, which is simply (4.24), also commutes.

The commutativity of (4.24) corresponds to equation

$$
\delta_{i j}\left(p_{0}^{*} \lambda_{i j}\right)\left(\delta_{j k}\right)=\nu_{i}\left(p_{0}^{*} g_{i j k}\right) \delta_{i k}\left(p_{0}^{*} g_{i j k}\right)^{-1},
$$

an equation whose terms are $G$-valued 2-forms on $U_{i j k}$. By the same reasoning as for (4.11), this can be written additively as

$$
\delta_{i j}+\lambda_{i j}\left(\delta_{j k}\right)-\lambda_{i j} \lambda_{j k}\left(\lambda_{i k}^{-1}\left(\delta_{i k}\right)\right)=\left[\nu_{i}, g_{i j k}\right],
$$

or, in the compact form of [5] (6.1.15), as

$$
\delta_{\lambda_{i j}}^{1}\left(\delta_{i j}\right)=\left[\nu_{i}, g_{i j k}\right] .
$$

Just we were able to derive (4.22) directly from (4.8) and the definitions (4.14) and (4.18), we now show that it is possible to deduce (4.26) from (4.18), (4.14) and (4.11). First of all,

$$
\begin{aligned}
\delta_{\lambda_{i j}}^{1}\left(\delta_{i j}\right) & =\delta_{\lambda_{i j}}^{1}\left(\delta_{\lambda_{i j}}^{0}\left(B_{i}\right)-\mathrm{d}_{m_{i}}^{1}\left(-\gamma_{i j}\right)\right) \\
& =\delta_{\lambda_{i j}}^{1} \delta_{\lambda_{i j}}^{0}\left(B_{i}\right)-\delta_{\lambda_{i j}}^{1} \mathrm{~d}_{m_{i}}^{1}\left(-\gamma_{i j}\right) .
\end{aligned}
$$

We now wish to assert that the Čech differential $\delta_{\lambda_{i j}}^{1}$ and de Rham differential $\mathrm{d}_{m_{i}}^{1}$ in (4.27) commute with each other, despite the fact that the 
1 -form $\gamma_{i j}$ takes its values in a non-commutative group $G$, and that $\mathrm{d}_{m_{i}}^{1}$ is not a homomorphism. For this we simplify our notation, by setting

$$
\widetilde{\gamma}_{i j}:=-\gamma_{i j} \in \mathfrak{g} \otimes \Omega_{U_{i j}}^{1}
$$

and

$$
\lambda_{i j k}:=\lambda_{i j} \lambda_{j k} \lambda_{i k}^{-1} \in \Gamma\left(U_{i j k}, \operatorname{Aut}\left(G_{i}\right)\right) .
$$

Equation (4.11) can be restated as

$$
\delta_{\lambda_{i j}}^{1} \widetilde{\gamma}:=\widetilde{\gamma}_{i j}+\lambda_{i j}\left(\widetilde{\gamma}_{j k}\right)-\lambda_{i j k}\left(\widetilde{\gamma}_{i k}\right)=-\mathrm{d} g_{i j k} g_{i j k}^{-1}-\left[m_{i}, g_{i j k}\right] .
$$

Lemma 4.3. The following equality between $G$-valued 2-forms above $U_{i j k}$ is satisfied:

$$
\mathrm{d}_{m_{i}}^{1} \delta_{\lambda_{i j}}^{1}\left(\widetilde{\gamma}_{i j}\right)=\delta_{\lambda_{i j}}^{1} \mathrm{~d}_{m_{i}}^{1}\left(\widetilde{\gamma}_{i j}\right) .
$$

Proof: We compute the left-hand side of the equation (4.30), taking into account the quadraticity equation (2.24)

$$
\begin{aligned}
& \mathrm{d}_{m_{i}}^{1} \delta_{\lambda_{i j}}^{1}\left(\widetilde{\gamma}_{i j}\right)=\mathrm{d}_{m_{i}}\left(\widetilde{\gamma}_{i j}\right)+\mathrm{d}_{m_{i}}^{1}\left(\lambda_{i j}\left(\widetilde{\gamma}_{j k}\right)\right)+\mathrm{d}_{m_{i}}^{1}\left(-\lambda_{i j k}\left(\widetilde{\gamma}_{i k}\right)\right)+ \\
&+\left[\widetilde{\gamma}_{i j}, \lambda_{i j}\left(\widetilde{\gamma}_{j k}\right)\right]-\left[\widetilde{\gamma}_{i j}, \lambda_{i j k}\left(\widetilde{\gamma}_{i k}\right)\right]--\left[\lambda_{i j}\left(\widetilde{\gamma}_{j k}\right), \lambda_{i j k}\left(\widetilde{\gamma}_{i k}\right)\right] \\
&=\mathrm{d}_{m_{i}}\left(\widetilde{\gamma}_{i j}\right)+\mathrm{d}_{m_{i}}^{1}\left(\lambda_{i j}\left(\widetilde{\gamma}_{j k}\right)\right)-\mathrm{d}_{m_{i}}^{1}\left(\lambda_{i j k}\left(\widetilde{\gamma}_{i k}\right)\right)+ \\
&+\left[\lambda_{i j k}\left(\widetilde{\gamma}_{i k}\right), \lambda_{i j k}\left(\widetilde{\gamma}_{i k}\right)\right]+\left[\widetilde{\gamma}_{i j}, \lambda_{i j}\left(\widetilde{\gamma}_{j k}\right)\right]- \\
&-\left[\widetilde{\gamma}_{i j}+\lambda_{i j}\left(\widetilde{\gamma}_{j k}\right), \lambda_{i j k}\left(\widetilde{\gamma}_{i k}\right)\right] .
\end{aligned}
$$

We now compute the right-hand side of (4.30):

$$
\delta_{\lambda_{i j}}^{1} \mathrm{~d}_{m_{i}}^{1}\left(\widetilde{\gamma}_{i j}\right)=\mathrm{d}_{m_{i}}^{1}\left(\widetilde{\gamma}_{i j}\right)+\lambda_{i j}\left(\mathrm{~d}_{m_{j}}^{1}\left(\widetilde{\gamma}_{j k}\right)\right)-\lambda_{i j k}\left(\mathrm{~d}_{m_{i}}^{1}\left(\widetilde{\gamma}_{i k}\right)\right) .
$$

By (4.7) and by the functoriality property (2.32), we find that

$$
\begin{aligned}
\lambda_{i j}\left(\mathrm{~d}_{m_{j}}^{1}\left(\widetilde{\gamma}_{j k}\right)\right) & =\mathrm{d}_{\lambda_{i j *} m_{j}}^{1}\left(\lambda_{i j}\left(\widetilde{\gamma}_{j k}\right)\right) \\
& =\mathrm{d}_{m_{i}}^{1}\left(\lambda_{i j}\left(\widetilde{\gamma}_{j k}\right)\right)+\left[\widetilde{\gamma}_{i j}, \lambda_{i j}\left(\widetilde{\gamma}_{j k}\right)\right]
\end{aligned}
$$

and by (2.34)

$$
\begin{aligned}
\lambda_{i j k}\left(\mathrm{~d}_{m_{i}}^{1}\left(\widetilde{\gamma}_{i k}\right)\right)= & \mathrm{d}_{\lambda_{i j k} m_{i}}^{1}\left(\lambda_{i j k}\left(\widetilde{\gamma}_{i k}\right)\right) \\
= & \mathrm{d}_{m_{i}}^{1}\left(\lambda_{i j k}\left(\widetilde{\gamma}_{i k}\right)\right)+\left[\left[\lambda_{i j k}, m_{i}\right], \lambda_{i j k}\left(\widetilde{\gamma}_{i k}\right)\right]+ \\
& +\left[\lambda_{i j k} \mathrm{~d} \lambda_{i j k}^{-1}, \lambda_{i j k}\left(\widetilde{\gamma}_{i k}\right)\right] .
\end{aligned}
$$

Inserting these expressions for $\lambda_{i j}\left(\mathrm{~d}_{m_{j}}^{1}\left(\widetilde{\gamma}_{j k}\right)\right)$ and $\lambda_{i j k}\left(\mathrm{~d}_{m_{i}}^{1}\left(\widetilde{\gamma}_{i k}\right)\right)$ into the righthand side of (4.31) we find the following expression for $\delta_{\lambda_{i j}}^{1} \mathrm{~d}_{m_{i}}^{1}\left(\widetilde{\gamma}_{i j}\right)$ : 


$$
\begin{array}{r}
\delta_{\lambda_{i j}}^{1} \mathrm{~d}_{m_{i}}^{1}\left(\widetilde{\gamma}_{i j}\right)=\mathrm{d}_{m_{i}}^{1}\left(\widetilde{\gamma}_{i j}\right)+\mathrm{d}_{m_{i}}^{1}\left(\lambda_{i j} \widetilde{\gamma}_{j k}\right)+\left[\widetilde{\gamma}_{i j}, \lambda_{i j}\left(\widetilde{\gamma}_{j k}\right)\right]- \\
-\mathrm{d}_{m_{i}}^{1}\left(\lambda_{i j k}\left(\widetilde{\gamma}_{i k}\right)-\left[\left[\lambda_{i j k}, m_{i}\right], \lambda_{i j k}\left(\widetilde{\gamma}_{i k}\right)\right]-\left[\lambda_{i j k} \mathrm{~d} \lambda_{i j k}^{-1}, \lambda_{i j k}\left(\widetilde{\gamma}_{i k}\right)\right]-\right. \\
-\mathrm{d}_{m_{i}}^{1}\left(\lambda_{i j k}\right)\left(\widetilde{\gamma}_{i k}\right)-\left[\lambda_{i j k} \mathrm{~d} \lambda_{i j k}^{-1}, \lambda_{i j k}\left(\widetilde{\gamma}_{i k}\right)\right] .
\end{array}
$$

Comparing this with the expression (4.31) for $\mathrm{d}_{m_{i}}^{1} \delta_{\lambda_{i j}}^{1}\left(\widetilde{\gamma}_{i j}\right)$, we see that the equation (4.30) is satisfied if and only if

$$
\begin{aligned}
{\left[\widetilde{\gamma}_{i j}+\lambda_{i j}\left(\widetilde{\gamma}_{j k}\right)-\lambda_{i j k}\left(\widetilde{\gamma}_{i k}\right), \lambda_{i j k}\left(\widetilde{\gamma}_{i k}\right)\right]=\left[\left[\lambda_{i j k}, m_{i}\right], \lambda_{i j k}\left(\widetilde{\gamma}_{i k}\right)\right]+} & \\
+ & {\left[\lambda_{i j k} \mathrm{~d} \lambda_{i j k}^{-1}, \lambda_{i j k}\left(\widetilde{\gamma}_{i k}\right)\right] . }
\end{aligned}
$$

By (2.9), this is simply a consequence of (4.29), since $\lambda_{i j k}=i\left(g_{i j k}\right)$.

We now return to our computation (4.27):

$$
\begin{array}{rlr}
\delta_{\lambda_{i j}}^{1}\left(\delta_{i j}\right) & =\delta_{\lambda_{i j}}^{1} \delta_{\lambda_{i j}}^{0}\left(B_{i}\right)-\delta_{\lambda_{i j}}^{1} \mathrm{~d}_{m_{i}}^{1}\left(-\gamma_{i j}\right) & \\
& =\delta_{\lambda_{i j}}^{1} \delta_{\lambda_{i j}}^{0}\left(B_{i}\right)-d_{m_{i}}^{1} \delta_{\lambda_{i j}}^{1}\left(-\gamma_{i j}\right) & \\
& =\left[g_{i j k}, B_{i}\right]-d_{m_{i}}^{1}\left(g_{i j k} d_{m_{i}}\left(g_{i j k}^{-1}\right)\right) & \\
& =\left[g_{i j k}, i_{B_{i}}-d m_{i}\right] & \text { by (2.27) } \\
& =\left[\nu_{i}, g_{i j k}\right] . &
\end{array}
$$

This finishes the second proof of equation (4.26) .

We now set

$$
\omega_{i}:=\mathrm{d}_{m_{i}}^{2}\left(B_{i}\right)
$$

Since the combinatorial definition of the twisted de Rham differential $\mathrm{d}^{2}$ (4] (3.3.1)) matches the global geometric definition (3.23) of the 3-curvature $\Omega$, this 3 -curvature $\Omega$ is locally described by the $G$-valued 3-forms $\omega_{i}$.

It follows from the definitions (4.14) and (4.32) of the forms $\nu_{i}$ and $\omega_{i}$, and from (2.26), that

$$
\begin{aligned}
\mathrm{d}_{m_{i}}^{3}\left(\omega_{i}\right) & =\mathrm{d}_{m_{i}}^{3} \mathrm{~d}_{m_{i}}^{2}\left(B_{i}\right) \\
& =\left[\mathrm{d}^{1} m_{i}, B_{i}\right] \\
& =\left[\nu_{i}, B_{i}\right]+\left[B_{i}, B_{i}\right]
\end{aligned}
$$

so that the local 3-curvature form $\omega_{i}$ satisfies the higher Bianchi identity

$$
\mathrm{d}_{m_{i}}^{3}\left(\omega_{i}\right)=\left[\nu_{i}, B_{i}\right] .
$$


A second relation between the forms $\nu_{i}$ and $\omega_{i}$ follows from their definitions and the Bianchi identity for the $\operatorname{Aut}(G)$-valued 1-form $m_{i}$ :

$$
\begin{aligned}
i\left(\omega_{i}\right) & =\mathrm{d}_{m_{i}}^{2} i\left(B_{i}\right) \\
& =\mathrm{d}_{m_{i}}^{2}\left(\mathrm{~d}^{1} m_{i}-\nu_{i}\right) \\
& =\mathrm{d}_{m_{i}}^{2}\left(-\nu_{i}\right),
\end{aligned}
$$

in other words

$$
\mathrm{d}_{m_{i}}^{2} \nu_{i}+i\left(\omega_{i}\right)=0 .
$$

This equation is the local form of equation(1.4), just as (4.33) was the local form of (1.5).

We will now show that the equation (4.18) for the 2 -forms $B_{i}$, which we write here as

$$
\delta_{\lambda_{i j}}^{0}\left(B_{i}\right)=\mathrm{d}_{m_{i}}^{1}\left(-\gamma_{i j}\right)+\delta_{i j},
$$

induces the corresponding gluing equation for the local 3 -forms $\omega_{i}$. From the definition of $\lambda_{i j}\left(\omega_{j}\right)$ and (2.33), it follows that

$$
\begin{aligned}
\lambda_{i j}\left(\omega_{j}\right) & =\lambda_{i j}\left(\mathrm{~d}_{m_{j}}^{2}\left(B_{j}\right)\right) \\
& =\mathrm{d}_{\lambda_{i j}{ }^{*} m_{j}}^{2} \lambda_{i j}\left(B_{j}\right)
\end{aligned}
$$

and by the gluing laws (4.8) and (4.18) for $m_{i}$ and $B_{i}$, this can be stated as

$$
\begin{aligned}
\lambda_{i j}\left(\omega_{j}\right) & =\mathrm{d}_{m_{i}-i\left(\gamma_{i j}\right)}^{2}\left(B_{i}+\delta_{i j}+\mathrm{d}_{m_{i}}^{1}\left(-\gamma_{i j}\right)\right) \\
& =\mathrm{d}_{m_{i}}^{2}\left(B_{i}\right)+\mathrm{d}_{m_{i}}^{2}\left(\delta_{i j}\right)+\mathrm{d}_{m_{i}}^{2} \mathrm{~d}_{m_{i}}^{1}\left(-\gamma_{i j}\right)-\left[\gamma_{i j}, B_{i}+\delta_{i j}+\mathrm{d}_{m_{i}}^{1}\left(-\gamma_{i j}\right)\right] .
\end{aligned}
$$

By (2.28), this last equality can be rewritten as

$$
\begin{aligned}
\lambda_{i j}\left(\omega_{j}\right) & =\omega_{i}+\mathrm{d}_{m_{i}}^{2}\left(\delta_{i j}\right)+\left[\mathrm{d}^{1} m_{i},-\gamma_{i j}\right]-\left[\gamma_{i j}, B_{i}\right]-\left[\gamma_{i j}, \delta_{i j}\right] \\
& =\omega_{i}+\mathrm{d}_{m_{i}}^{2}\left(\delta_{i j}\right)+\left[\gamma_{i j}, \mathrm{~d}^{1} m_{i}-B_{i}\right]-\left[\gamma_{i j}, \delta_{i j}\right]
\end{aligned}
$$

and by (4.21) this proves the gluing law for the 3 -forms $\omega_{i}[5]$ (6.1.23):

$$
\lambda_{i j}\left(\omega_{j}\right)=\omega_{i}+\mathrm{d}_{m_{i}}^{2}\left(\delta_{i j}\right)+\left[\gamma_{i j}, \nu_{i}\right]-\left[\gamma_{i j}, \delta_{i j}\right] .
$$

By combining this with the gluing law (4.22) for $\nu_{i}$, we see that (4.35) can finally be rewritten in the more compact form

$$
\lambda_{i j}\left(\omega_{j}\right)+\left[{ }^{\lambda_{i j}} \nu_{j}, \gamma_{i j}\right]=\omega_{i}+\mathrm{d}_{m_{i}}^{2}\left(\delta_{i j}\right)
$$




\section{5 Čech-de Rham coboundaries}

\section{1}

We saw in section 2 how a change in the choice trivializing data $\left(x_{i}, \phi_{i j}\right)$ in a gerbe $\mathcal{P}$ could be measured by a pair $\left(r_{i}, \theta_{i j}\right)$ (3.10), (3.11) inducing a coboundary relation (3.15) between the corresponding cocycle pairs $\left(\lambda_{i j}, g_{i j k}\right)$. We will now examine how the terms $\left(m_{i}, \gamma_{i j}\right),\left(\nu_{i}, \delta_{i j}\right)$ and $B_{i}$ introduced in section 4 vary when the arrows $\gamma_{i}$ (4.1) and $\delta_{i}$ (4.12) which determine them have been modified.

The difference between the arrow $\gamma_{i}$ and an analogous arrow $\gamma_{i}^{\prime}$ is measured by a 1 -form $e_{i} \in \operatorname{Lie}(G) \otimes \Omega_{U_{i}}^{1}$, defined by the commutativity of the following diagram:

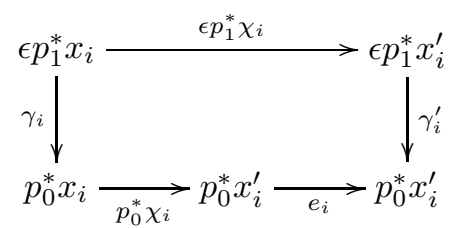

This conjugates to a commutative diagram

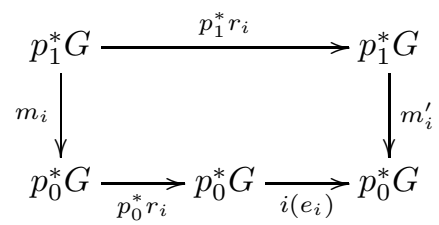

so that

$$
\begin{aligned}
m_{i}^{\prime} & =i\left(e_{i}\right)\left(p_{0}^{*} r_{i}\right) m_{i}\left(p_{1}^{*} r_{i}\right)^{-1} \\
& =i\left(e_{i}\right)\left[p_{0}^{*} r_{i} m_{i} p_{0}^{*} r_{i}^{-1}\right]\left[p_{0}^{*} r_{i} p_{1}^{*} r_{i}{ }^{-1}\right]
\end{aligned}
$$

In classical terms, this is expressed as an equation

$$
\begin{aligned}
m_{i}^{\prime} & ={ }^{r_{i}} m_{i}+r_{i} \mathrm{~d} r_{i}^{-1}+i\left(e_{i}\right) \\
& ={ }^{r_{i} *} m_{i}+i\left(e_{i}\right) .
\end{aligned}
$$

which compares the connections $m_{i}$ and $m_{i}^{\prime}$ induced on the group $G$ by the arrows $\gamma_{i}$ and $\gamma_{i}^{\prime}$. 
We now consider the following diagram in $\mathcal{P}_{U_{i j}}$ :

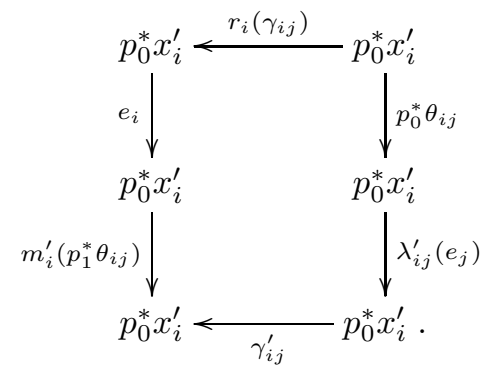

Proposition 5.1. The diagram (5.4) is commutative.

Proof: Consider the diagram

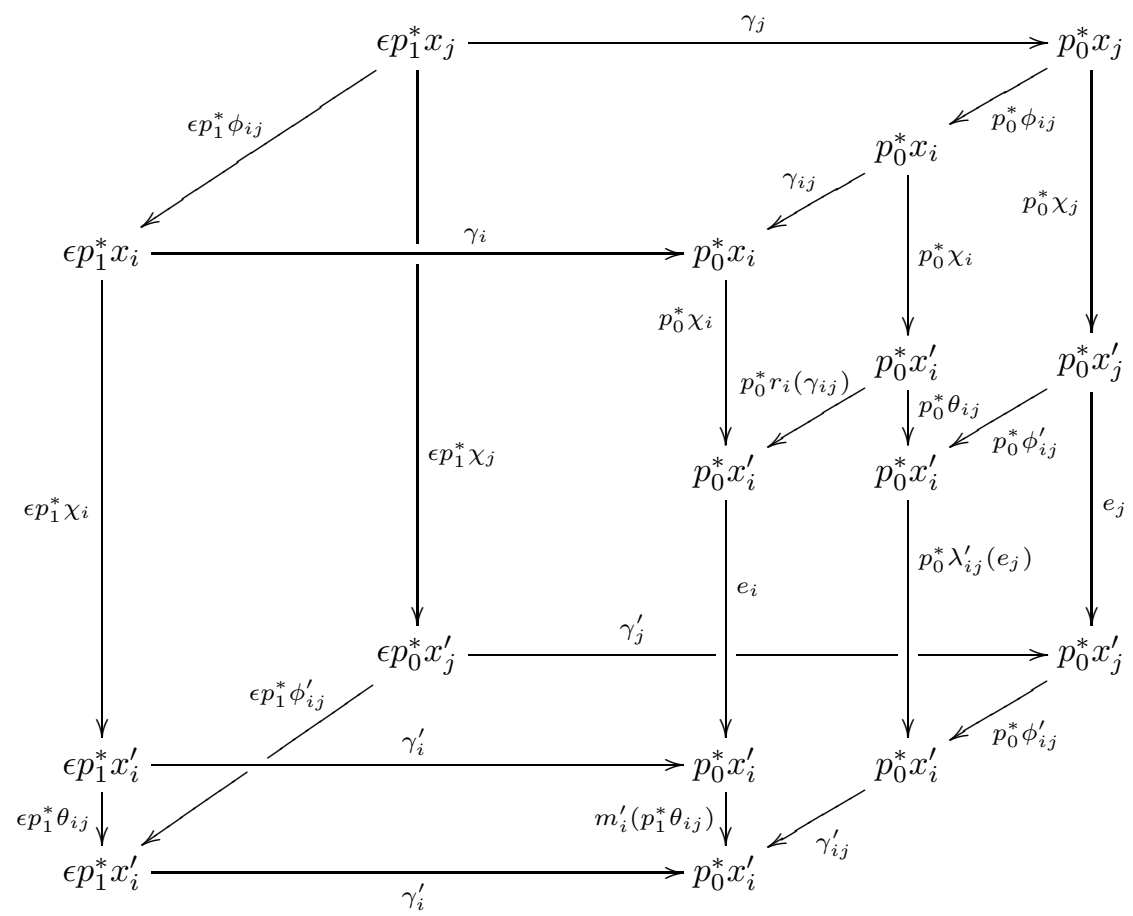

The lower front square of the right-hand face of this cube is just the square (5.4). Since we know that all the other squares in this diagram commute, so does the square (5.4).

The commutativity of (5.4) is equivalent to the equation

$$
m_{i}^{\prime}\left(p_{1}^{*} \theta_{i j}\right) e_{i} r_{i}\left(\gamma_{i j}\right)=\gamma_{i j}^{\prime} \lambda_{i j}^{\prime}\left(e_{j}\right) p_{0}^{*} \theta_{i j} .
$$


This may be rewritten in classical notation as:

$$
\left(\gamma_{i j}^{\prime}-{ }^{\theta_{i j}} r_{i}\left(\gamma_{i j}\right)\right)+\left(\lambda_{i j}^{\prime}\left(e_{j}\right)-{ }^{\theta_{i j}} e_{i}\right)=\mathrm{d}_{m_{i}^{\prime}} \theta_{i j} \theta_{i j}^{-1} .
$$

We now choose a family of arrows $\delta_{i}^{\prime}: \kappa p_{0}^{*} x_{i}^{\prime} \longrightarrow p_{0}^{*} x_{i}^{\prime}$. The families $\delta_{i}^{\prime}$ and $\gamma_{i}^{\prime}$ determine as in (4.13) a family of $\mathfrak{g}$-valued 2-form $B_{i}^{\prime}$ above $U_{i}$. The latter in turn determines, together with the pair of form $\left(m_{i}^{\prime}, \gamma_{i j}^{\prime}\right)$ (5.2), (5.7), a new pair of 2-forms $\left(\nu_{i}^{\prime}, \delta_{i j}^{\prime}\right)$ and a 3 -form $\omega_{i}^{\prime}$ satisfying the corresponding equations (4.22), (4.34), (4.26), (4.33) and (4.35). The families $\delta_{i}$ and $\delta_{i}^{\prime}$ are compared by the following analogue of diagram (5.1):

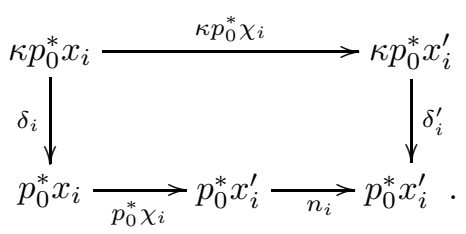

We will now compare the 2 -forms $B_{i}$ and $B_{i}^{\prime}$. We consider the diagram

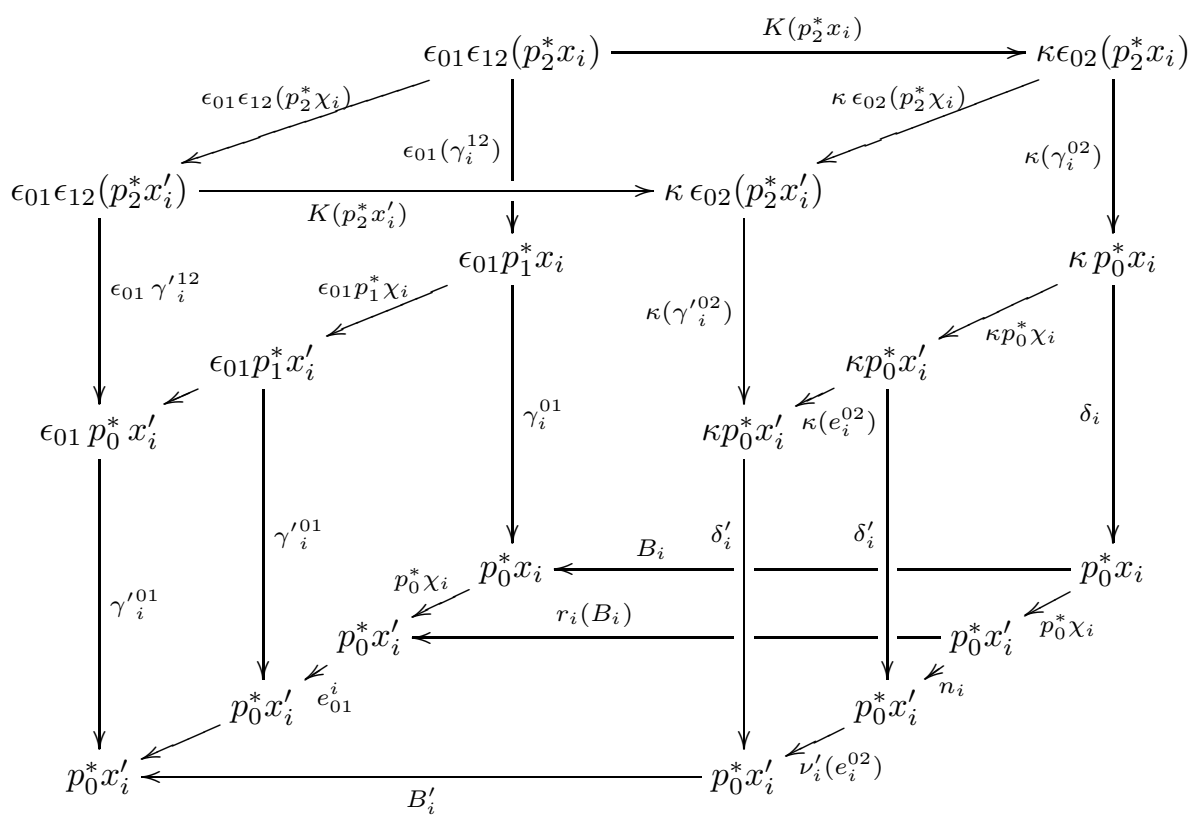

in which the upper and lower unlabelled arrows are respectively $\epsilon_{01}\left(p_{1}^{*} e_{i}^{12}\right)$ and $m_{i}^{\prime 01}\left(e_{i}^{12}\right)$. 
The front square (or rather hexagon) of the bottom face

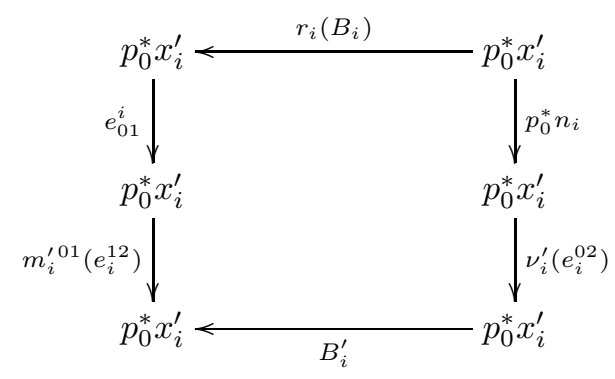

is commutative, since all other squares in diagram (5.9) are. Equivalently, since the action of the $\operatorname{Aut}(G)$-valued 2-form $\nu_{i}^{\prime}$ on $e_{i}^{02}$ is trivial, this proves that the equation

$$
B_{i}^{\prime}=r_{i}\left(B_{i}\right)-\mathrm{d}_{m_{i}^{\prime}}^{1}\left(-e_{i}\right)-n_{i} .
$$

is satisfied. In particular for given $B_{i}$ and $e_{i}$, the 2 -forms $B_{i}^{\prime}$ and $n_{i}$ actually determine each other.

By conjugation, diagram (5.8) induces a commutative diagram

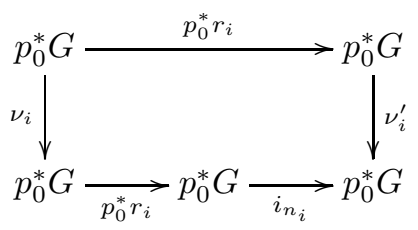

equivalent to the equation

$$
i\left(n_{i}\right) p_{0}^{*} r_{i} \nu_{i}=\nu_{i}^{\prime} p_{0}^{*} r_{i}
$$

In classical terms, this is the simpler analogue

$$
\nu_{i}^{\prime}={ }^{r_{i}} \nu_{i}+i\left(n_{i}\right)
$$

for $\nu_{i}$ of the equation (5.2) for $m_{i}$.

We will now show that this coboundary equation for $\nu_{i}$ can be derived from the definition (4.14) of $\nu_{i}$, and the coboundary equations (5.2) and (5.10) for $m_{i}$ and $B_{i}$ :

$$
\begin{aligned}
\nu_{i}^{\prime} & =\mathrm{d}^{1} m_{i}^{\prime}-i\left(B_{i}^{\prime}\right) \\
& =\mathrm{d}^{1}\left({ }^{r_{i} *} m_{i}+i\left(e_{i}\right)\right)-i\left(r_{i}\left(B_{i}\right)+n_{i}+\mathrm{d}_{m_{i}^{\prime}}^{1}\left(-e_{i}\right)\right) \\
& ={ }^{r_{i}} \mathrm{~d}^{1} m_{i}+i\left(\mathrm{~d}^{1} e_{i}\right)+\left[{ }^{r_{i} *} m_{i}, i\left(e_{i}\right)\right]-i\left(r_{i}\left(B_{i}\right)\right)+i\left(\mathrm{~d}_{m_{i}^{\prime}}^{1}\left(-e_{i}\right)\right)+i\left(n_{i}\right) \\
& ={ }^{r_{i}}\left(\mathrm{~d}^{1} m_{i}-i\left(B_{i}\right)\right)+i\left(n_{i}\right)+i\left(\mathrm{~d}_{m_{i}^{\prime}}^{1}\left(-e_{i}\right)+\mathrm{d}^{1} e_{i}+\left[{ }^{r_{i}^{*}} m_{i}, e_{i}\right]\right)
\end{aligned}
$$


In order to prove (5.11), it now suffices to verify that the 3 terms in the last summand of the final equation cancel each other out:

$$
\begin{aligned}
\mathrm{d}_{m_{i}^{\prime}}^{1}\left(-e_{i}\right)+\mathrm{d}^{1}\left(e_{i}\right)+\left[{ }^{r_{i} *} m_{i}, e_{i}\right] & =\mathrm{d}^{1}\left(-e_{i}\right)-\left[m_{i}^{\prime}, e_{i}\right]+\mathrm{d}^{1} e_{i}+\left[{ }^{r_{i} *} m_{i}, e_{i}\right] \\
& =\mathrm{d}^{1}\left(-e_{i}\right)+\mathrm{d}^{1} e_{i}-\left[e_{i}, e_{i}\right] \\
& =0 .
\end{aligned}
$$

The other equation satisfied by the forms $n_{i}$ is the counterpart of equation (5.6). It is obtained by considering the following diagram, analogous to (5.5):

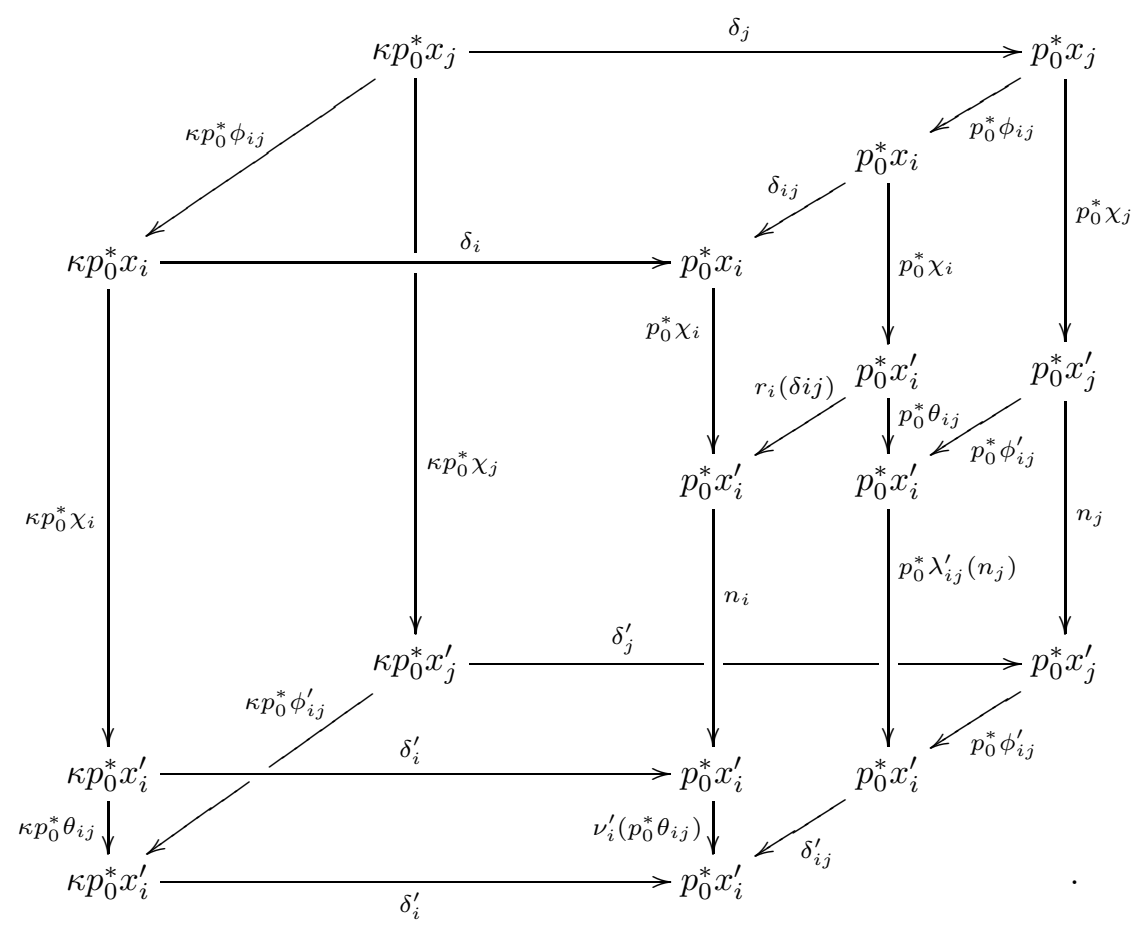

The lower front square on the right-hand face

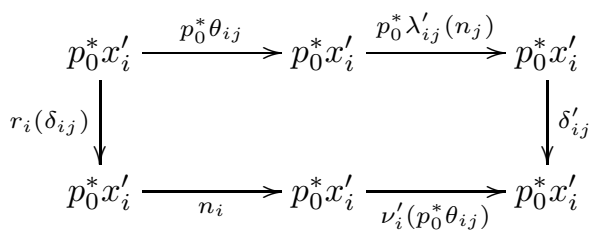

of diagram (5.12) is commutative, since all other squares in this diagram are. 
This proves that equation

$$
\nu_{i}^{\prime}\left(p_{0}^{*} \theta_{i j}\right) n_{i} r_{i}\left(\delta_{i j}\right)=\delta_{i j}^{\prime} p_{0}^{*} \lambda_{i j}^{\prime}\left(n_{j}\right) p_{0}^{*} \theta_{i j}
$$

in Lie $(G) \otimes \Omega_{U_{i} / S}^{2}$ is satisfied. Regrouping the various terms in this equation as we did above for equation (5.6), we find that it is equivalent, in additive notation, to

$$
\left(\delta_{i j}^{\prime}-r_{i}\left(\delta_{i j}\right)\right)+\left(\lambda_{i j}^{\prime}\left(n_{j}\right)-{ }^{\theta_{i j}} n_{i}\right)=\left[\nu_{i}^{\prime}, \theta_{i j}\right],
$$

an equation for 2 -forms very similar to equation (5.7) for 1 -forms.

We will now examine the effect of the chosen transfomations

$$
\left(\lambda_{i j}, g_{i j k}, m_{i}, \gamma_{i j}\right) \quad \longrightarrow \quad\left(\lambda_{i j}^{\prime}, g_{i j k}^{\prime}, m_{i}^{\prime}, \gamma_{i j}^{\prime}\right)
$$

and $B_{i} \longrightarrow B_{i}^{\prime}$ (5.10) on the 3 -curvature 3 -forms $\omega_{i}$ (4.32). For this, it will be convenient to set

$$
\bar{e}_{i}:=r_{i}^{-1}\left(e_{i}\right) \quad \text { and } \quad \bar{n}_{i}:=r_{i}^{-1}\left(n_{i}\right) .
$$

It follows from (2.23), (2.10), and the transformation formula (5.3) that

$$
\mathrm{d}_{m_{i}^{\prime}}^{n}\left(r_{i}(\eta)\right)=r_{i}\left(\mathrm{~d}_{m_{i}}^{n}(\eta)+\left[\bar{e}_{i}, \eta\right]\right)
$$

for any $G$-valued $n$-form $\eta$ with $n>1$. In particular

$$
\begin{aligned}
\mathrm{d}_{m_{i}^{\prime}}^{1}\left(-e_{i}\right) & =\mathrm{d}_{r_{i} * m_{i}}^{1}\left(-e_{i}\right)-\left[e_{i}, e_{i}\right] \\
& =r_{i}\left(\mathrm{~d}_{m_{i}}^{1}\left(-\bar{e}_{i}\right)-\left[\bar{e}_{i}, \bar{e}_{i}\right]\right)
\end{aligned}
$$

so that (5.10) may be expressed as

$$
B_{i}^{\prime}=r_{i}\left(B_{i}-\mathrm{d}_{m_{i}}^{1}\left(-\bar{e}_{i}\right)+\left[\bar{e}_{i}, \bar{e}_{i}\right]-\bar{n}_{i}\right) .
$$

Applying once more the formula (5.14), we find that

$$
\begin{aligned}
\omega_{i}^{\prime}= & \mathrm{d}_{m_{i}^{\prime}}^{2}\left(B_{i}^{\prime}\right) \\
= & \mathrm{d}_{m_{i}^{\prime}}^{2}\left(r_{i}\left(B_{i}-\mathrm{d}_{m_{i}}^{1}\left(-\bar{e}_{i}\right)+\left[\bar{e}_{i}, \bar{e}_{i}\right]-\bar{n}_{i}\right)\right) \\
= & r_{i}\left(\mathrm{~d}_{m_{i}}^{2}\left(B_{i}-\mathrm{d}_{m_{i}}^{1}\left(-\bar{e}_{i}\right)+\left[\bar{e}_{i}, \bar{e}_{i}\right]-\bar{n}_{i}\right)\right)+ \\
& \quad+\left[\bar{e}_{i}, B_{i}-\mathrm{d}_{m_{i}}^{1}\left(-\bar{e}_{i}\right)+\left[\bar{e}_{i}, \bar{e}_{i}\right]-\bar{n}_{i}\right] .
\end{aligned}
$$

We now make use of (2.28) in order to compute the value of the expression $\mathrm{d}_{m_{i}}^{2} \mathrm{~d}_{m_{i}}^{1}\left(-\bar{e}_{i}\right)$ which arises when we expand the first summand of the last equation (5.15):

$$
\begin{aligned}
\mathrm{d}_{m_{i}}^{2} \mathrm{~d}_{m_{i}}^{1}\left(-\bar{e}_{i}\right) & =\left[\mathrm{d}^{1} m_{i},-\bar{e}_{i}\right]+\left[\mathrm{d}^{1}\left(-\bar{e}_{i}\right),-\bar{e}_{i}\right]+\left[\left[m_{i},-\bar{e}_{i}\right],-\bar{e}_{i}\right] \\
& =-\left[\mathrm{d}^{1} m_{i}, \bar{e}_{i}\right]+\left[\mathrm{d}^{1} \bar{e}_{i}, \bar{e}_{i}\right]+\left[\left[m_{i}, \bar{e}_{i}\right], \bar{e}_{i}\right] .
\end{aligned}
$$


Inserting this expression into (5.15), we find that

$$
\begin{aligned}
\omega_{i}^{\prime}=r_{i}\left(\omega_{i}+\left[\mathrm{d}^{1} m_{i}, \bar{e}_{i}\right]-\left[\mathrm{d}^{1} \bar{e}_{i}, \bar{e}_{i}\right]-\left[\left[m_{i}, \bar{e}_{i}\right], \bar{e}_{i}\right]-\mathrm{d}_{m_{i}}^{2}\left(\bar{n}_{i}\right)+\right. \\
\left.+\mathrm{d}_{m_{i}}^{2}\left[\bar{e}_{i}, \bar{e}_{i}\right]+\left[\bar{e}_{i}, B_{i}\right]-\left[\bar{e}_{i}, \mathrm{~d}_{m_{i}}^{1}\left(-\bar{e}_{i}\right)\right]-\left[\bar{e}_{i}, \bar{n}_{i}\right]\right) .
\end{aligned}
$$

The four terms

$$
-\left[\mathrm{d}^{1} \bar{e}_{i}, \bar{e}_{i}\right]-\left[\left[m_{i}, \bar{e}_{i}\right], \bar{e}_{i}\right]+\mathrm{d}_{m_{i}}^{2}\left[\bar{e}_{i}, \bar{e}_{i}\right]-\left[\bar{e}_{i}, \mathrm{~d}_{m_{i}}^{1}\left(-\bar{e}_{i}\right)\right]
$$

cancel each other out, so that we are left in (5.16) with

$$
\begin{aligned}
\omega_{i}^{\prime} & =r_{i}\left(\omega_{i}+\left[\mathrm{d}^{1} m_{i}, \bar{e}_{i}\right]-\mathrm{d}_{m_{i}}^{2}\left(\bar{n}_{i}\right)+\left[\bar{e}_{i}, B_{i}\right]-\left[\bar{e}_{i}, \bar{n}_{i}\right]\right) \\
& =r_{i}\left(\omega_{i}+\left[\mathrm{d}^{1} m_{i}-i\left(B_{i}\right), \bar{e}_{i}\right]+\left[\bar{n}_{i}, \bar{e}_{i}\right]-\mathrm{d}_{m_{i}}^{2}\left(\bar{n}_{i}\right)\right) \\
& =r_{i}\left(\omega_{i}\right)+r_{i}\left(\left[\nu_{i}, \bar{e}_{i}\right]\right)+r_{i}\left(\left[\bar{n}_{i}, \bar{e}_{i}\right]\right)-r_{i}\left(\mathrm{~d}_{m_{i}}^{2}\left(\bar{n}_{i}\right)\right) \\
& =r_{i}\left(\omega_{i}\right)+\left[{ }^{r_{i}} \nu_{i}, e_{i}\right]+\left[n_{i}, e_{i}\right]-\mathrm{d}_{r_{i} m_{i}}^{2}\left(n_{i}\right)
\end{aligned}
$$

where in the last line we made use of the functoriality property (2.10) of the bracket operation. Amalgamating the last two summands, we may finally write the coboundary transformation for the 3 -curvature form $\omega_{i}$ in the compact form

$$
\omega_{i}^{\prime}=r_{i}\left(\omega_{i}\right)+\left[{ }^{r_{i}} \nu_{i}, e_{i}\right]-\mathrm{d}_{m_{i}^{\prime}}^{2}\left(n_{i}\right) .
$$

If instead we amalgamate the second and third term in (5.17), we find the equivalent formulation

$$
\omega_{i}^{\prime}=r_{i}\left(\omega_{i}\right)+\left[\nu_{i}^{\prime}, e_{i}\right]-\mathrm{d}_{r_{i} * m_{i}}^{2}\left(n_{i}\right)
$$

\section{Remark 5.1 (Comparison with [5]):}

The coboundary equation (5.18) is compatible with equation (6.2.19) of 5], but neither is a special case of the other. Here we allowed both the trivializing data $\left(x_{i}, \phi_{i j}\right)$ for the gerbe and the expressions $\left(\gamma_{i}, \delta_{i}, B_{i}\right)$ for the curving data to vary, whereas in the coboundary equations of [5] the gerbe data $\left(x_{i}, \phi_{i j}\right)$ was fixed and only the $\left(\gamma_{i}, \delta_{i}, B_{i}\right)$ varied. This restriction amounted to setting $\left(r_{i}, \theta_{i j}\right)=(1,1)$ in our equation (5.7). On the other hand, a notion of equivalence between cocycles was introduced in [5] which was more extensive than the one considered here. In order for these to be comparable, one must suppose that the arrow $h$ in diagram (4.2.1) of [5] is the identity map, i.e. that the pair of differential forms $\left(\pi_{i}, \eta_{i j}\right)$ associated to $h$ in loc. cit $\S 6.2$ is trivial. This is a reasonable assumption, since a non-trivial arrow $h$ could really be termed a gauge transformation, rather than a coboundary term. With 
this additional condition, the last two summands in equation (6.2.19) of [5] vanish, so that this equation reduces to

$$
\omega_{i}^{\prime}=\omega_{i}+\delta_{m_{i}}^{2}\left(\alpha_{i}\right)-\left[\nu_{i}^{\prime}, E_{i}\right] .
$$

This simplified equation is compatible with our equation (5.18) with $r_{i}=1$, under the correspondence $e_{i}:=-E_{i}$ and $n_{i}:=-\alpha_{i}$.

\section{References}

1. Aschieri, P., Cantini, L., Jurčo B.: Nonabelian Bundle Gerbes, their Differential Geometry and Gauge Theory, Comm. in Math. Phys. 254, 367-400 (2005).

2. Baez J. C. and Schreiber U.: Higher Gauge Theory. Preprint, arXiv:math/0511710

3. Breen, L.: Classification of 2-gerbes and 2-stacks Astérisque 225, Société Mathématique de France (1994)

4. Breen, L., Messing, W.: Combinatorial Differential Forms. Advances in Math. 164, 203-282 (2001)

5. Breen, L., Messing, W.: Differential Geometry of Gerbes. Advances in Math. 198, 732-846 (2005)

6. Breen, L.: Notes on 1- and 2-gerbes: arXiv:math/0611317, to appear in the Proceedings of the IMA Meeting " $n$-Categories: Foundations and Applications" (Minneapolis, 2004)

7. Brylinski, J.-L.: Loop spaces, characteristic classes and geometric quantization. Progress in Math. 107, Birkhäuser, Boston, Basel, Berlin (1993)

8. Hitchin, N.: Lectures on special lagrangian submanifolds, preprint arXiv:math.DG/9907034 (1999).

9. Kobayashi S., Nomizu K.: Foundations of differential geometry, Interscience Tracts in Pure and Applied Mathematics 15 (1969).

10. Kock, A.: Differential forms with values in groups. Bull. Austral. Math. Soc 25, 357-386 (1982)

11. Kock, A.: Combinatorics of curvature, and the Bianchi identity. Theory and Applications of Categories 2, 69-89 (1996).

12. Laurent-Gengoux C., Stenion M., Xu P.: Non Abelian Differential Gerbes. Preprint, arXiv:math.DG/0511696

13. Loday J.-L.: The diagonal of the Stasheff polytope. This volume, preprint arXiv:0710.0572,

14. Murray, M. K.: Bundle Gerbes. J. of the London Math. Society 54, 403-416 (1996).

15. Saneblidze S., Umble, R.: A diagonal on the Associahedra. Preprint, arXiv:math.AT/0011065

16. Schreiber U.: From Loop Space Mechanics to Nonabelian Strings. Preprint, hep-th/0509163

17. Stasheff J.: Homotopy associativity of $H$-spaces I. Trans. Amer. Math. Soc.108, 275-292 (1963).

18. Ulbrich, K. - H.: On cocycle bitorsors and gerbes over a Grothendieck topos. Math. Proc. Cambridge Phil. Soc. 110, 49-55 (1991) 